\title{
Spin modular categories
}

Beliakova, Anna ; Blanchet, Christian ; Contreras, Eva

\begin{abstract}
Modular categories are a well-known source of quantum 3-manifold invariants. In this paper we study structures on modular categories which allow to define refinements of quantum 3-manifold invariants involving cohomology classes or generalized spin and complex spin structures. A crucial role in our construction is played by objects which are invertible under tensor product. All known examples of cohomological or spin type renements of the Witten-Reshetikhin-Turaev 3-manifold invariants are special cases of our construction. In addition, we establish a splitting formula for the refined invariants, generalizing the well-known product decomposition of quantum invariants into projective ones and those determined by the linking matrix.
\end{abstract}

DOI: https://doi.org/10.4171/QT/95

Posted at the Zurich Open Repository and Archive, University of Zurich

ZORA URL: https://doi.org/10.5167/uzh-141265

Journal Article

Originally published at:

Beliakova, Anna; Blanchet, Christian; Contreras, Eva (2017). Spin modular categories. Quantum Topology, 8(3):459-504.

DOI: https://doi.org/10.4171/QT/95 


\title{
SPIN MODULAR CATEGORIES
}

\author{
ANNA BELIAKOVA, CHRISTIAN BLANCHET, AND EVA CONTRERAS
}

\begin{abstract}
Modular categories are a well-known source of quantum 3-manifold invariants. In this paper we study structures on modular categories which allow to define refinements of quantum 3-manifold invariants involving cohomology classes or generalized spin and complex spin structures. A crucial role in our construction is played by objects which are invertible under tensor product. All known examples of cohomological or spin type refinements of the Witten-Reshetikhin-Turaev 3-manifold invariants are special cases of our construction. In addition, we establish a splitting formula for the refined invariants, generalizing the well-known product decomposition of quantum invariants into projective ones and those determined by the linking matrix.
\end{abstract}

\section{INTRODUCTION}

In the late 80's, inspired by Witten's ideas [22], Reshetikhin and Turaev [18] came up with a construction of new 3-manifold invariants, known as WRT quantum invariants. Few years later, Turaev [20] formalized this construction by introducing the notion of modular category. His main result is that any modular category gives rise to a quantum 3-manifold invariant.

A modular category is a special kind of ribbon category which has a finite set of simple objects $\Gamma$, including the unit object $\mathbf{1} \in \Gamma$, satisfying duality, domination and non-degeneracy axioms. A ribbon category is a monoidal category with braiding, twist and compatible duality. Ribbon categories are universal receivers for invariants of ribbon graphs [20]. Examples of modular categories arise from representation theory of quantum groups, when the quantum parameter $q$ is a root of unity, or can be constructed skein theoretically [5, 11, 3, 1]. Many authors [9], [19], [2] observed independently that for some special values of $q$, the $\mathfrak{s l}_{2}$ WRT invariants admit spin and cohomological refinements. Cohomological refinements give rise to homotopy quantum field theories (HQFT's), constructed by Turaev in [21]. However, spin refinements do not fit in the framework of HQFT's.

The main aim of this paper is to provide an algebraic setting for spin type refinements of quantum 3-manifold invariants. Before explaining our results let us recall few definitions.

Given a group $G$, a $G$-category was defined in [21, Section VI] as an additive monoidal category $\mathcal{C}$ with left duality and unit object 1 that splits as a disjoint union of full subcategories $\left\{\mathcal{C}_{\alpha}\right\}_{\alpha \in G}$ such that

- $\operatorname{Hom}_{\mathcal{C}}\left(\mathcal{C}_{\alpha}, \mathcal{C}_{\beta}\right)=0$ if $\alpha \neq \beta$;

- for $U \in \mathcal{C}_{\alpha}$ and $V \in \mathcal{C}_{\beta}, U \otimes V \in \mathcal{C}_{\alpha \beta}$;

- $\mathbf{1} \in \mathcal{C}_{1}$, and for $U \in \mathcal{C}_{\alpha}, U^{*} \in \mathcal{C}_{\alpha^{-1}}$.

Date: November 18, 2014. 
We call an object $t$ of a modular category invertible if there exists an object $\lambda$, such that $t \otimes \lambda \cong \mathbf{1}$. It is easy to see that isomorphism classes of invertible objects form a finite abelian group under tensor multiplication. Let us denote by $G=G_{\mathcal{C}}$ the group of isomorphism classes of invertible objects in the modular category $\mathcal{C}$. In Section 5 we show that the braiding (or monodromy) coefficients of $\lambda \in \Gamma$ with $t \in G$ define a map $\Gamma \rightarrow \operatorname{Hom}\left(G, S^{1}\right)=\widehat{G}$. This map induces on $\mathcal{C}$ the structure of a $\widehat{G}$-category.

Note the braiding matrix defines a bilinear form on $G$, and the twist coefficients extend it to a quadratic form. A special role in our approach will play a subgroup $H$ of $G$, such that the bilinear form restricted to $H$ is trivial while the quadratic extension is not.

Let us state our main definition.

Definition. Let $\mathcal{C}$ be a modular category with a group $G$ of invertible objects. For any subgroup $H \subset G$, we call $\mathcal{C} H$-refinable if $H \subset \mathrm{Ob}\left(\mathcal{C}_{1}\right)$. Moreover, an $H$-refinable modular category $\mathcal{C}$ is called $H$-spin if the twist quadratic form restricted to $H$ is non-trivial, or equivalently if $H$ has at least one element with twist coefficient -1 . When $H$ is cyclic of order $d$, we will use shorthand $d$-spin and $d$-refinable.

For example, the $\mathfrak{s l}_{2}$ modular category at the $r$ th root of unity $q$ is 2-refinable for $r=0$ $(\bmod 2)$ and it is $2-$ spin if $r=0(\bmod 4)$. The group $G=H=\mathbb{Z} / 2 \mathbb{Z}$ is generated by the $(r-1)$-dimensional representation.

We say that $t \in G$ has order $n$ if $n$ is the minimal integer such that $t^{n} \cong 1$. We will see that the order of an element with twist -1 has to be even.

$H$-refinable modular categories which are non spin give rise to invariants of pairs $(M, h)$ for any compact orientable 3 -manifold $M$ and a cohomology class $h \in H^{1}(M ; \widehat{H})$. They also fit in the setting of modular group-categories introduced in [21]. In the spin case, the formalism of [21] does not strictly apply since the subcategory in trivial degree is not modular.

Let us concentrate on the spin case. If we have a $H$-spin modular category, then the twist coefficients define an order 2 element $v \in \widehat{H}$, which we will call the spin character. We will extend the definition of generalized spin structures with modulo $d$ coefficients given in [2] to this situation and define $(\widehat{H}, v)$ generalized spin structures. One of the results of this paper is the following.

Theorem 1. Any $H$-spin modular category $\mathcal{C}$ with associated spin character $v \in \operatorname{Tor}_{2}(\widehat{H})$ provides a topological invariant $\tau_{\mathcal{C}}(M, \sigma)$ of a pair $(M, \sigma)$, where $\sigma$ is a $(\widehat{H}, v)$ generalized spin structure on $M$. Moreover,

$$
\tau_{\mathcal{C}}(M)=\sum_{\sigma} \tau_{\mathcal{C}}(M, \sigma)
$$

We expect that Theorem 1 extends naturally to a spin type TQFT.

In Section 2 we define $d$-complex spin structures. Let us denote the set of such structures on $M$ by $\operatorname{Spin}_{d}^{c}(M)$. Generalizing results of [7], we identify $\operatorname{Spin}_{d}^{c}(M)$ with the set of modulo $d$ Chern vectors which are further used for constructing extensions of WRT invariants.

Theorem 2. Suppose $d$ is an even positive integer. For any $2 d$-spin modular category $\mathcal{C}$, there exists a topological invariant $\tau_{\mathcal{C}}(M, \sigma)$ of a pair $(M, \sigma)$, where $\sigma \in \operatorname{Spin}_{d}^{c}(M)$ is a d-complex spin structure on $M$. 
We call a modular category $\mathcal{C}$ reduced if it is $G_{\mathcal{C}}$-refinable.

Assume the group of invertible objects $G=\langle t\rangle$ of our modular category $\mathcal{C}$ is cyclic, but $t \in \operatorname{Ob}\left(\mathcal{C}_{\delta}\right)$ with $\delta \neq 1$. If $|G|=d$, then there is a positive integer $m$, such that $\delta m=d$. Clearly, $\mathcal{C}$ is not reduced, but $H$-refinable, where the subgroup $H$ of order $\delta$ is generated by $t^{m} \in \mathcal{C}_{1}$.

Given these data, one way to construct refined invariants is by using the $\delta$-refinable structure on $\mathcal{C}$. In Section 7 we show that there is a more efficient way to compute this invariant. Namely, there always exists a smaller reduced category $\tilde{\mathcal{C}}$, which leads to the same invariant up to a correction term fully determined by the linking matrix. If $\operatorname{gcd}(m, \delta)=1, \tilde{\mathcal{C}}$ is particularly simple and coincides with $\mathcal{C}_{1}$ where $1 \in \widehat{H}$.

Theorem 3. Let $\mathcal{C}$ be a modular category with a cyclic group of invertible objects $G=\langle t\rangle$. Assume $d=|G|, t \in \mathcal{C}_{\delta}$ and $\delta m=d$. Then, there exists a reduced $\delta$-refinable category $\widetilde{\mathcal{C}}, a$ positive integer $\alpha$ and a root of unity $\xi$ such that for any closed oriented 3-manifold $M$ we have

$$
\tau_{\mathcal{C}}(M, \sigma)=\alpha^{-b_{1}(M)} \tau_{\widetilde{\mathcal{C}}}(M, \sigma) \tau_{\xi}^{\mathrm{MMO}}(M, \sigma)
$$

where $\tau_{\xi}^{\mathrm{MMO}}(M, \sigma)$ is the refined Murakami-Ohtsuki-Okada invariant. We have either $\sigma \in$ $H^{1}\left(M, \mathbb{Z}_{\delta}\right)$ or $\sigma \in \operatorname{Spin}_{\delta}(M)$, and $b_{1}(M)$ is the first Betti number.

In the particular case, when $\operatorname{gcd}(\delta, m)=1$, we have $\alpha=1, \xi$ is a root of unity of order $m$ and $\tau_{\xi}^{\mathrm{MMO}}(M, \sigma)$ does not depend on $\sigma$ so that we have

$$
\tau_{\mathcal{C}}(M)=\tau_{\tilde{\mathcal{C}}}(M) \tau_{\xi}^{\mathrm{MMO}}(M) .
$$

The Murakami-Ohtsuki-Okada invariant defined in [16] depends only on the homological information which can be obtained from the linking matrix of the surgery link.

Theorem 3 generalizes the well-known decompositon results for quantum invariants stated in $[10,2]$. We expect that these decomposition results extend to refined TQFTs.

Organization of the paper. After recalling the basic definitions of $\mathrm{Spin}^{c}$-structures, we give homotopy theoretical and also combinatorial descriptions of their reduction modulo $d$. Further we recall the definitions of ribbon and modular categories and of quantum 3-manifold invariants. Section 5 deals with modular categories containing invertible objects. The refined invariants are studied in Sections 6 and 7. The last section is devoted to the proof of Theorem 3.

\section{Complex SPIN StRuCtures MOD $d$}

In this section, given a compact orientable 3-manifold $M$, we define the set $\operatorname{Spin}_{d}^{c}(M)$.

Throughout this paper all manifolds are assumed to be compact and oriented; all (co)homology groups are computed with integer coefficients, unless otherwise is specified; $\mathbb{Z}_{x}$ denotes the cyclic group of integers modulo $x$.

2.1. Spin ${ }^{c}$-structures. Complex spin structures are additional structures some manifolds can be endowed with and just like the more common spin structures, they can be seen as a generalization of orientations. We recall basic facts and equivalent ways to define $\operatorname{Spin}^{c}$-structures following the lines of [7] and [17]. 
Let $n \geq 1$ be an integer. The group $\operatorname{Spin}(n)$ is defined as the non-trivial double cover of the special orthogonal group $\mathrm{SO}(n)$ :

$$
1 \longrightarrow \mathbb{Z}_{2} \longrightarrow \operatorname{Spin}(n) \stackrel{\lambda}{\longrightarrow} \mathrm{SO}(n) \longrightarrow 1 \text {. }
$$

Example. $\operatorname{Spin}(1) \cong \mathbb{Z}_{2}, \operatorname{Spin}(2) \cong S^{1}$ and $\operatorname{Spin}(3) \cong \mathrm{SU}(2)$.

The complex spin group is defined as the quotient

$$
\operatorname{Spin}^{c}(n):=\frac{\operatorname{Spin}(n) \times S^{1}}{\mathbb{Z}_{2}}
$$

where $\mathbb{Z}_{2}$ is generated by $(-1,-1) \in \operatorname{Spin}(n) \times S^{1}$. It follows that the map $\rho: \operatorname{Spin}^{c}(n) \rightarrow \operatorname{SO}(n)$, defined as $\rho([A, z])=\lambda(A)$ is a principal $S^{1}$-fibration.

Example. $\operatorname{Spin}^{c}(3) \cong \mathrm{U}(2)$.

Let $X$ be an $n$-dimensional Riemannian manifold and denote by $P_{\mathrm{SO}(n)} \rightarrow X$ the principal bundle of oriented orthonormal frames of $X$.

Definition ([17]). A Spin ${ }^{c}$-structure on the manifold $X$ is a principal $\operatorname{Spin}^{c}(n)-$ bundle $P_{\operatorname{Spin}^{c}(n)} \stackrel{s}{\longrightarrow}$ $X$, together with a map $\pi: P_{\operatorname{Spin}^{c}(n)} \longrightarrow P_{\mathrm{SO}(n)}$ that restricted to the fibers is $\rho$, i.e, makes the following diagram commute, i.e,

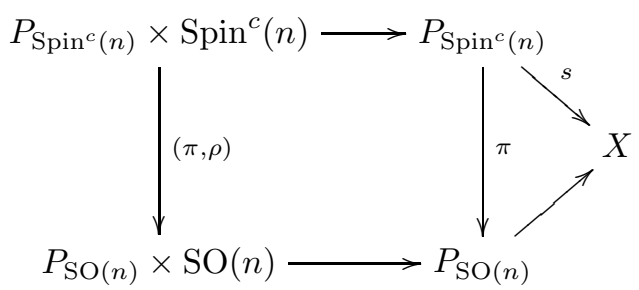

Since $\rho: \operatorname{Spin}^{c}(n) \rightarrow \mathrm{SO}(n)$ is the $S^{1}$-bundle of the unique non trivial line bundle over $\mathrm{SO}(n)$ ([14]) and isomorphism classes of principal $S^{1}$-bundles over $X$ are in one-to-one correspondence with elements of $H^{2}(X ; \mathbb{Z})$, complex spin structures can be defined as cohomology classes:

Alternative Definition ([17]). A $\operatorname{Spin}^{c}$-structure on the manifold $X$ is an element $\sigma \in H^{2}\left(P_{\mathrm{SO}(n)} ; \mathbb{Z}\right)$ whose restriction to every fiber of $P_{\mathrm{SO}(n)} \longrightarrow X$ is the unique non trivial element of $H^{2}(\mathrm{SO}(n) ; \mathbb{Z}) \cong$ $\mathbb{Z}_{2}$, i.e,

$$
\operatorname{Spin}^{c}(X)=\left\{\sigma \in H^{2}\left(P_{\mathrm{SO}(n)} ; \mathbb{Z}\right) \mid \sigma_{\mid \text {fiber }} \neq 0 \in H^{2}(\mathrm{SO}(n) ; \mathbb{Z})\right\} .
$$

Let $\operatorname{BSO}(n)$ denote the Grassman manifold of oriented $n$-planes in $\mathbb{R}^{\infty}$ and let $\gamma_{\mathrm{SO}(n)}$ be the universal $n$-dimensional oriented vector bundle over $\operatorname{BSO}(n)$. Note that for discrete topological groups $G$, the classifying space $B G$ is Eilenberg-Maclane of type $(G, 1)$. Let $h$ be the unique (up to homotopy) non-homotopically trivial map from $\operatorname{BSO}(n)$ to the Eilenberg-Maclane space $\mathrm{K}(\mathbb{Z}, 3)$. We fix $h$ in its homotopy class and we define the fibration $\operatorname{BSpin}^{c}(n) \stackrel{\pi}{\rightarrow} \mathrm{BSO}(n)$ as the pull-back under $h$ of the path-space fibration over $\mathrm{K}(\mathbb{Z}, 3)$. If we set $\gamma_{\operatorname{Spin}^{c}(n)}:=\pi^{*}\left(\gamma_{\mathrm{SO}(n)}\right)$, then:

Alternative Definition. A $\operatorname{Spin}^{c}$-structure on the manifold $X$ is a homotopy class of bundle maps between the (stable) tangent bundle TX of $X$ and $\gamma_{\operatorname{Spin}^{c}(n)}$. 
2.1.1. The Chern map. Let us denote by $\alpha: \operatorname{Spin}^{c}(n) \rightarrow S^{1}$ the homomorphism $\alpha([A, z])=z^{2}$. Then, to any $\operatorname{Spin}^{c}$ - structure $\sigma$ on an $n$-dimensional manifold $X$ (in the sense of Definition 2.1), one can associate a complex line bundle as follows: using the map $\alpha$, the action of the group $\operatorname{Sppin}^{c}(n)$ on the space $P_{\operatorname{Spin}^{c}(n)}$ extends to an action on the product $P_{\operatorname{Spin}^{c}(n)} \times \mathbb{C}$ and we consider its orbit space

$$
\operatorname{det}(\sigma):=\left(P_{\operatorname{Spin}^{c}(n)} \times \mathbb{C}\right) / \operatorname{Spin}^{c}(n),
$$

called the determinant line bundle of $\sigma$. The Chern map

$$
c: \operatorname{Spin}^{c}(X) \rightarrow H^{2}(X)
$$

is defined as $c(\sigma):=c_{1}(\operatorname{det}(\sigma))$, where $c_{1}$ is the first Chern class of the bundle $\operatorname{det}(\sigma)$, and it is affine over the doubling map $H^{2}(X) \stackrel{\cdot 2}{\rightarrow} H^{2}(X)$. See [7] for more details.

2.2. $d$-complex spin structures. In this subsection we define $d$-complex spin structures (short $\operatorname{Spin}_{d}^{c}$-structures) on $n$-dimensional manifolds and we describe some of their properties. Then, we focus on dimension three and we present a set of refined Kirby's moves for $\operatorname{Spin}_{d}^{c}$-manifolds obtained by surgery along links in $S^{3}$.

Let $\beta: H^{2}\left(\mathrm{BSO}(n) ; \mathbb{Z}_{2}\right) \longrightarrow H^{3}\left(\mathrm{BSO}(n) ; \mathbb{Z}_{d}\right)$ be the Bockstein homomorphism associated to the exact sequence of groups:

$$
0 \longrightarrow \mathbb{Z}_{d} \stackrel{\cdot 2}{\longrightarrow} \mathbb{Z}_{2 d} \longrightarrow \mathbb{Z}_{2} \longrightarrow 0
$$

The following lemma will help justify our construction of $\operatorname{Spin}_{d}^{c}$-structures.

Lemma. The group $H^{3}\left(\operatorname{BSO}(n) ; \mathbb{Z}_{d}\right)$ is cyclic of order 2 generated by $\beta\left(w_{2}\right)$, where $w_{2}$ is the second Stiefel-Whitney class.

Since the elements of the group $H^{3}\left(\mathrm{BSO}(n) ; \mathbb{Z}_{d}\right) \cong \mathbb{Z}_{2}$ are in one-to-one correspondence with homotopy classes of maps in $\left[\mathrm{BSO}(n) ; \mathrm{K}\left(\mathbb{Z}_{d}, 3\right)\right]$, there is a unique (up to homotopy) nonhomotopically trivial map $g: \operatorname{BSO}(n) \longrightarrow \mathrm{K}\left(\mathbb{Z}_{d}, 3\right)$. We fix $g$ in its homotopy class and define the fibration $\pi_{d}: \operatorname{BSpin}_{d}^{c}(n) \longrightarrow \mathrm{BSO}(n)$ as the pull-back of the path space fibration of $\mathrm{K}\left(\mathbb{Z}_{d}, 3\right)$ under the map $g$ :

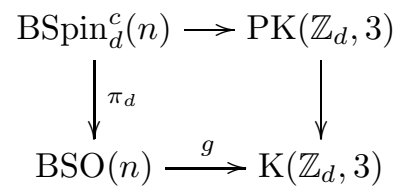

We set $\gamma_{\operatorname{Spin}_{d}^{c}(n)}=\pi_{d}^{*}\left(\gamma_{\mathrm{SO}(n)}\right)$. Note that another choice of the map $g$ in its homotopy class yields

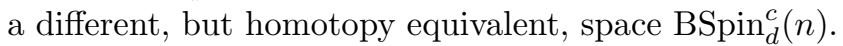

Let $X$ be an $n$-dimensional Riemannian manifold.

Definition. A $d$-complex spin structure on $X$ is a homotopy class of a bundle map between the (stable) tangent bundle TX of $X$ and $\gamma_{\operatorname{Spin}_{d}^{c}(n)}$.

Alternative Definition. A $d$-complex spin structure on $X$ is a homotopy class of a lift $\bar{f}$ of $f$ to $\operatorname{BSpin}_{d}^{c}(n)$, where $f: X \longrightarrow \operatorname{BSO}(n)$ is a classifying map for the bundle TX. 


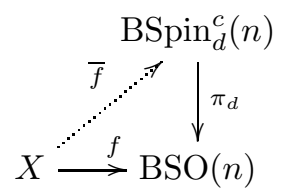

Since the fiber of $\pi_{d}$ is the Eilenberg-Maclane space $\mathrm{K}\left(\mathbb{Z}_{d}, 2\right)$, there is a unique obstruction $w_{X}$ to the existence of lifts $\bar{f}$ and this obstruction lies in the group $H^{3}\left(X ; \mathbb{Z}_{d}\right)$.

Note that the universal obstruction $w \in H^{3}\left(\operatorname{BSO}(n) ; \mathbb{Z}_{d}\right)$ (obtained from $w_{X}$ by setting $X=$ $\mathrm{BSO}(n)$ and $\left.f=\operatorname{id}_{\mathrm{BSO}(n)}\right)$ is non-zero, therefore not all manifolds can admit $\operatorname{Spin}_{d}^{c}$-structures. To see this, assume the contrary. Then, the fibration $\pi_{d}: \operatorname{BSpin}_{d}^{c}(n) \longrightarrow \operatorname{BSO}(n)$ has a section $s$ and the map $k \circ s$ lifts $g$ to the contractible space $\operatorname{PK}\left(\mathbb{Z}_{d}, 3\right)$. Therefore $g$ must be null-homotopic which contradicts the choice we made for $g$.

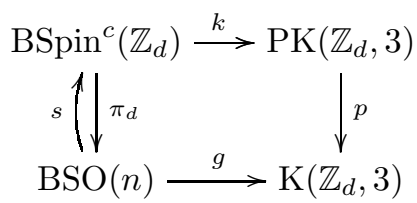

As a consequence, the universal obstruction $w$ is the generator $\beta\left(w_{2}\right)$ of $H^{3}\left(\mathrm{BSO}(n) ; \mathbb{Z}_{d}\right)$.

Proposition. The set $\operatorname{Spin}_{d}^{c}(X)$ of $d$-complex spin structures on a manifold $X$ is non-empty, if and only if, $\beta\left(w_{2}(X)\right)=0 \in H^{3}\left(X ; \mathbb{Z}_{d}\right)$. If non-empty, the set $\operatorname{Spin}_{d}^{c}(X)$ is affine over $H^{2}\left(X ; \mathbb{Z}_{d}\right)$ $\left(w_{2}(X)\right.$ is the second Stiefel-Whitney class of $\left.X\right)$.

Proof.

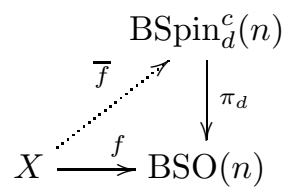

Let $f$ be the classifying map of the (stable) tangent bundle of $X$. Then, the lifts $\bar{f}$ of $f$ to $\operatorname{BSpin}^{c}\left(\mathbb{Z}_{d}\right)$ are in one-to-one correspondence with the sections of the bundle $f^{*}\left(\gamma_{\operatorname{Spin}_{d}^{c}(n)}\right)$. The obstruction $w_{X}$ to the existence of such sections is a characteristic cohomology class so by its naturality property we have

$$
\begin{aligned}
w_{X}\left(f^{*}\left(\gamma_{\operatorname{Spin}_{d}^{c}(n)}\right)\right) & =f^{*}\left(w\left(\gamma_{\operatorname{Spin}_{d}^{c}(n)}\right)\right) \\
& =f^{*}\left(\beta\left(w_{2}\right)\right) .
\end{aligned}
$$

The above relation together with the naturality of Bockstein homomorphisms imply the result. The second part of the theorem follows by standard arguments of obstruction theory.

2.2.1. Restriction to the boundary. Let us consider a manifold $X$ with boundary. Then, any section of $\left.T X\right|_{\partial X}$ transverse to $\partial X$ and oriented outwards gives rise to a homotopy class of isomorphisms between the oriented vector bundles $\mathbb{R} \oplus T \partial X$ and $\left.T X\right|_{\partial X}$. Therefore, there is a well-defined restriction map

$$
\operatorname{Spin}_{d}^{c}(X) \rightarrow \operatorname{Spin}_{d}^{c}(\partial X)
$$

affine over the map $H^{2}\left(X ; \mathbb{Z}_{d}\right) \stackrel{i^{*}}{\rightarrow} H^{2}\left(\partial X ; \mathbb{Z}_{d}\right)$ induced by the inclusion $i: \partial X \rightarrow X$. 
2.2.2. From $\operatorname{Spin}^{c}$ to $\operatorname{Spin}_{d}^{c}$. We have seen in Section 2.1 that the fibration $\operatorname{BSpin}^{c}(n) \stackrel{\pi}{\rightarrow} \operatorname{BSO}(n)$ is defined as the pull-back under $h$ of the path-space fibration over $\mathrm{K}(\mathbb{Z}, 3)$. The modulo $d$ restriction morphism $\xi: \mathbb{Z} \rightarrow \mathbb{Z}_{d}$ induces a map $\mathrm{K}(\mathbb{Z}, 3) \stackrel{\xi_{*}}{\rightarrow} \mathrm{K}\left(\mathbb{Z}_{d}, 3\right)$ on the level of EilenbergMaclane spaces and further, via $\xi_{*}$, a natural map $\operatorname{BSpin}^{c}(n) \rightarrow \operatorname{BSpin}_{d}^{c}(n)$. As a result, there exists a well-defined natural map

$$
\operatorname{Spin}^{c}(X) \rightarrow \operatorname{Spin}_{d}^{c}(X)
$$

affine over $H^{2}(X) \stackrel{\xi^{*}}{\rightarrow} H^{2}\left(X ; \mathbb{Z}_{d}\right)$ induced by $\xi$.

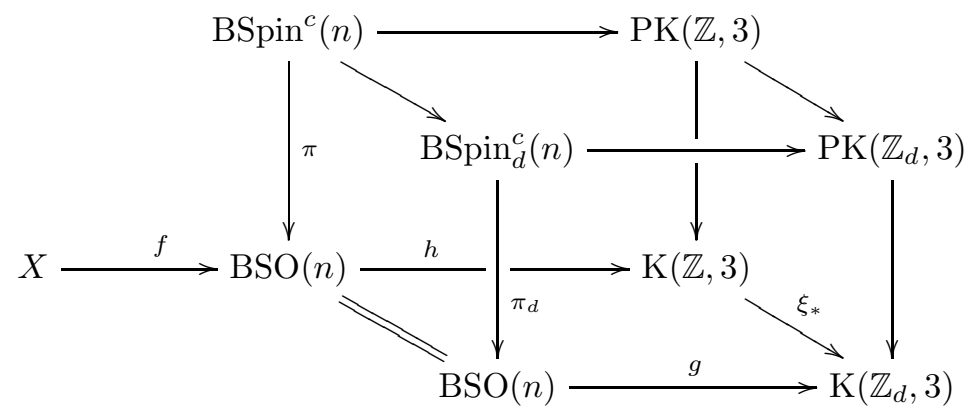

2.3. Combinatorial description of $\operatorname{Spin}_{d}^{c}$-structures. Let $L=\left(L_{1}, \cdots, L_{n}\right)$ be an oriented framed link in $S^{3}$ with linking matrix $\left(L_{i j}\right)_{i, j=1, n}$ and denote by $S^{3}(L)$ the 3-manifold obtained by surgery. The manifold $S^{3}(L)$ is the boundary of a 4 -manifold $W_{L}$, constructed from a 4 -ball $D^{4}$ by attaching $n 2$-handles $\left(D^{2} \times D^{2}\right)_{i}$ along embeddings of $-\left(S^{1} \times D^{2}\right)_{i}$ in concordance with the orientation and framing of each component $L_{i}$. The 4 -manifold $W_{L}$ is sometimes called trace of surgery.

Let us define the set

$$
\mathcal{S}_{d}^{c}(L)=\frac{\left\{\left(\sigma_{1}, \ldots, \sigma_{n}\right) \in\left(\mathbb{Z}_{2 d}\right)^{n} \mid \sigma_{i}=L_{i i} \quad(\bmod 2)\right\}}{2 \operatorname{Im} L}
$$

whose elements will be called modulo d Chern vectors.

Theorem. There is a canonical bijection

$$
\operatorname{Spin}_{d}^{c}\left(S^{3}(L)\right) \stackrel{\phi_{L}}{\longrightarrow} \mathcal{S}_{d}^{c}(L) .
$$

Proof. We have seen in the previous section that $\operatorname{Spin}^{c}$-structures induce $d$-complex spin structures. In particular, the map $r_{d}: \operatorname{Spin}^{c}\left(W_{L}\right) \longrightarrow \operatorname{Spin}_{d}^{c}\left(W_{L}\right)$ is surjective since it is affine over the surjective map $H^{2}\left(W_{L} ; \mathbb{Z}\right) \longrightarrow H^{2}\left(W_{L} ; \mathbb{Z}_{d}\right)$ induced by restriction modulo $d$ of coefficients and similarly, the restriction map $r: \operatorname{Spin}_{d}^{c}\left(W_{L}\right) \longrightarrow \operatorname{Spin}_{d}^{c}\left(S^{3}(L)\right)$ is surjective since it is affine over the surjective map $H^{2}\left(W_{L} ; \mathbb{Z}_{d}\right) \longrightarrow H^{2}\left(S^{3}(L) ; \mathbb{Z}_{d}\right)$ induced by inclusion.

With the help of $r$ and $r_{d}$, we define the map $\phi_{L}: \operatorname{Spin}_{d}^{c}\left(S^{3}(L)\right) \longrightarrow \mathcal{S}_{d}^{c}(L)$ as follows: any $\sigma \in \operatorname{Spin}_{d}^{c}\left(S^{3}(L)\right)$ is the image of an element $\tilde{\sigma} \in \operatorname{Spin}^{c}\left(W_{L}\right)$ under the composition $r \circ r_{d}$. The value $c(\tilde{\sigma}) \in H^{2}\left(W_{L} ; \mathbb{Z}\right) \cong \mathbb{Z}^{n}$ is characteristic for $L$ (see [7]), therefore $c(\tilde{\sigma})=\left(c_{1}, \ldots, c_{n}\right) \in \mathbb{Z}^{n}$ with $c_{i}=L_{i i}(\bmod 2)$. We set $\phi_{L}(\sigma)$ to be the image of $c(\tilde{\sigma})(\bmod 2 d)$ in $\mathcal{S}_{d}^{c}(L)$.

- $\phi_{L}(\sigma)$ is well-defined: let us assume that $\left(r \circ r_{d}\right)^{-1}(\sigma)$ contains two different elements $\tilde{\sigma_{1}}$ and $\tilde{\sigma_{2}}$. Then, they differ by $y \in H^{2}\left(W_{L}\right) \cong \mathbb{Z}^{n}$ whose modulo $d$ reduction belongs to $\operatorname{Im} L$ in $\left(\mathbb{Z}_{d}\right)^{n}$. Since $2 y(\bmod 2 d)$ belongs to $2 \operatorname{Im} L$ in $\left(\mathbb{Z}_{2 d}\right)^{n}$, we get that $\left[c\left(\tilde{\sigma_{2}}\right)\right]=$ 
$\left[c\left(\tilde{\sigma_{1}}\right)+2 y\right]=\left[c\left(\tilde{\sigma_{1}}\right)\right]$ (the first equality follows from the fact that the Chern map is affine over the doubling map).

- $\phi_{L}$ is injective: let us assume that $\phi_{L}\left(\sigma_{1}\right)=\phi_{L}\left(\sigma_{2}\right)$. Then, the preimages $\tilde{\sigma_{1}}$ and $\tilde{\sigma_{2}}$ (of $\sigma_{1}$ and $\sigma_{2}$, respectively) under $r \circ r_{d}$ differ by $y \in H^{2}\left(W_{L}\right) \cong \mathbb{Z}^{n}$ such that $2 y(\bmod 2 d)$ belongs to $2 \operatorname{Im} L$ in $\left(\mathbb{Z}_{2 d}\right)^{n}$. This implies that $y(\bmod d)$ belongs to $\operatorname{Im} L$ in $\left(\mathbb{Z}_{d}\right)^{n}$ and therefore $\sigma_{1}=\sigma_{2}$.

The sets $\operatorname{Spin}_{d}^{c}\left(S^{3}(L)\right)$ and $\mathcal{S}_{d}^{c}(L)$ have the same cardinality, hence $\phi_{L}$ is bijective.

From now on, we will reffer to the set $\mathcal{S}_{d}^{c}(L)$ as the combinatorial description of $d$-complex spin structures on the surgered manifold $S^{3}(L)$.

2.4. $\operatorname{Spin}_{d}^{c}$ Kirby moves. A celebrated theorem of Kirby [8] states that two (oriented) framed links in $S^{3}$ produce the same manifold by surgery, if and only if, they are related by a finite sequence of local geometric transformations called Kirby moves. In what follows, we present a refined version of the original Kirby theorem for manifolds equipped with $\operatorname{Spin}_{d}^{c}$-structures.

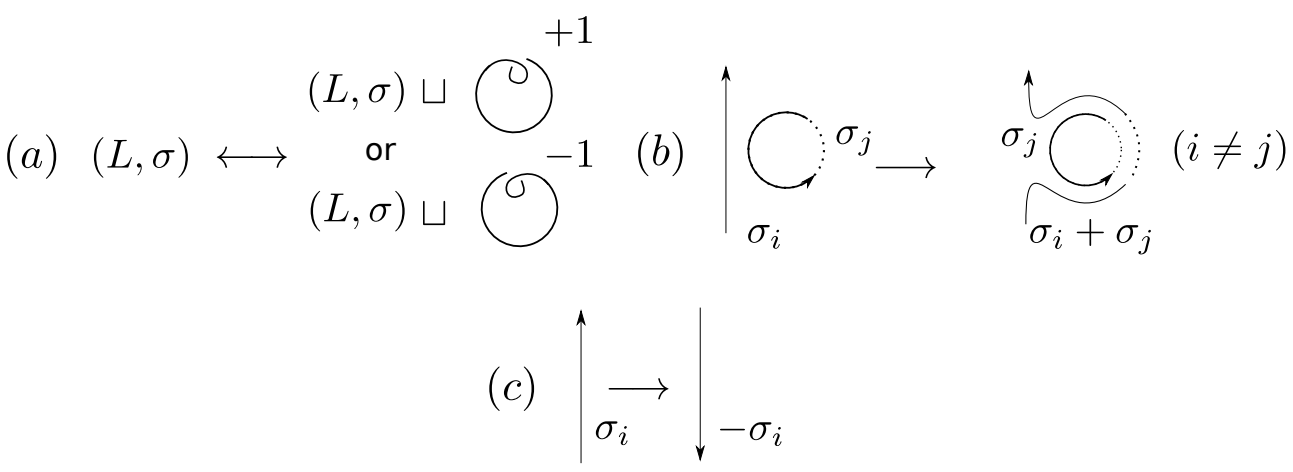

Figure 2.1. Refined $\operatorname{Spin}_{d}^{c}$-Kirby moves (a) Stabilization (b) Handle slide (c) Orientation reversal. Note that we use the blackboard framing and the labels refer to modulo $d$ Chern vectors.

Theorem. Let $(L, \sigma)$ and $\left(L^{\prime}, \sigma^{\prime}\right)$ be two oriented framed links with Chern vectors $\sigma, \sigma^{\prime}$. Then, the manifolds $\left(S^{3}(L), \sigma\right)$ and $\left(S^{3}\left(L^{\prime}\right), \sigma^{\prime}\right)$ are $\operatorname{Spin}_{d}^{c}$-homeomorphic, if and only if, the pairs $(L, \sigma)$ and $\left(L^{\prime}, \sigma^{\prime}\right)$ are related by a finite sequence of the moves in Figure 2.1 or their inverses.

Proof. We must check that for any of the Kirby moves (stabilization, handle slide and orientation reversal) $L \stackrel{K}{\rightarrow} L^{\prime}$, the Chern vectors change under the homeomorphism $S^{3}(L) \rightarrow S^{3}\left(L^{\prime}\right)$ in the way described by Figure 2.1. To do this, note that the map $r \circ r_{d}: \operatorname{Spin}^{c}\left(W_{L}\right) \rightarrow \operatorname{Spin}_{d}^{c}\left(S^{3}(L)\right)$ defined in the proof of Theorem 2.3 is surjective and the following diagram commutes:

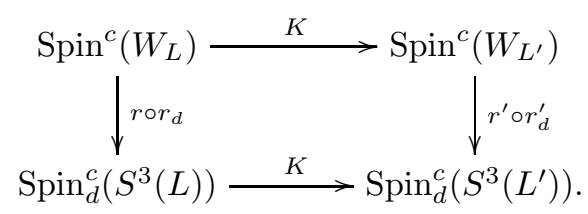


Since $\operatorname{Spin}^{c}$-structures on $W_{L}$ are combinatorially described as the elements of the set

$$
\left\{\left(c_{1}, \cdots, c_{n}\right) \in \mathbb{Z}^{n} \mid c_{i}=L_{i i} \quad(\bmod 2)\right\}
$$

and their change under Kirby moves is known ([7]), the conclusion follows.

\section{Generalized spin Structures}

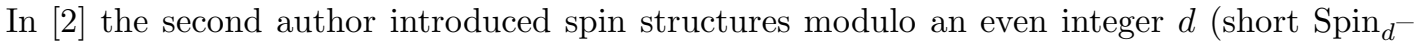
structures). In dimension three, he gave a combinatorial description of such structures as well as a refined set of Kirby moves. In this section we recall his results using the notations of Section 2.3 and extend the definition to a possibly non cyclic group of coefficient $K$ with distinguished order 2 element $v \in K$.

3.1. Combinatorial description of $\operatorname{Spin}_{d}$-structures. Consider the set

$$
\mathcal{S}_{d}(L)=\left\{\left(s_{1}, \ldots, s_{n}\right) \in\left(\mathbb{Z}_{d}\right)^{n} \mid \sum_{j=1}^{n} L_{i j} s_{j}=\frac{d}{2} L_{i i} \quad(\bmod d)\right\} .
$$

The elements of $\mathcal{S}_{d}(L)$ are called modulo d characteristic solutions of $L$.

Lemma. There is a canonical bijection

$$
\operatorname{Spin}_{d}\left(S^{3}(L)\right) \stackrel{\psi_{L}}{\longrightarrow} \mathcal{S}_{d}(L) .
$$

Proof. Given a $\operatorname{Spin}_{d}$-structure $\sigma$ on $S^{3}(L)$ it can be extended to $W_{L}$ if and only if a certain cohomology class in $H^{2}\left(W_{L}, S^{3}(L) ; \mathbb{Z}_{d}\right)$ vanishes. We denote this class by $w\left(W_{L} ; \sigma\right)$ and we call it relative obstruction. To any $\sigma \in \operatorname{Spin}_{d}\left(S^{3}(L)\right)$ there is associated a relative obstruction $w\left(W_{L} ; \sigma\right)$ in $H^{2}\left(W_{L}, S^{3}(L) ; \mathbb{Z}_{d}\right)$. Since the group $H^{2}\left(W_{L}, S^{3}(L) ; \mathbb{Z}_{d}\right)$ is free of rank $n$, taking the coefficients of the relative obstruction we obtain a map $\psi_{L}: \operatorname{Spin}_{d}\left(S^{3}(L)\right) \longrightarrow\left(\mathbb{Z}_{d}\right)^{n}$. We will show that this map is injective and its image coincides with $\mathcal{S}_{d}(L)$. Let us consider the embedding $\eta: \mathbb{Z}_{2} \hookrightarrow \mathbb{Z}_{d}$. Then, the relative obstruction $w\left(W_{L} ; \sigma\right)=\eta_{*}\left(w_{2}\left(W_{L}\right)\right)$, where $\eta_{*}$ is the induced map on the level of cohomology. Given an integral 2-cycle $x$, we denote by $x \cdot x$ its self-intersection number and by $[x]_{m}$ its modulo $m$ restriction. The second Stiefel-Whitney class $w_{2}\left(W_{L}\right)$ is defined by the following equation:

$$
\left\langle w_{2}\left(W_{L}\right),[x]_{2}\right\rangle=x \cdot x \quad(\bmod 2), \forall x
$$

therefore, the relative obstruction is defined by

$$
\left\langle w\left(W_{L} ; \sigma\right),[x]_{d}\right\rangle=\frac{d}{2} x \cdot x \quad(\bmod d), \forall x .
$$

Using functoriality and writing $w\left(W_{L} ; \sigma\right)$ in the preferred basis of $H^{2}\left(W_{L} ; \mathbb{Z}_{d}\right)$, the result follows.

The set $\mathcal{S}_{d}(L)$ will be referred to as a combinatorial description of $\operatorname{Spin}_{d}$-structures on the surgered manifold $S^{3}(L)$. 
3.2. $(K, v)$-spin structures. Let $K$ be a finite abelian group and $v$ a non trivial element in the 2 -torsion subgroup $\operatorname{Tor}_{2}(K)$, then we can define $(K, v)$ generalized spin structures.

Definition. For $n \geq 3$, an $(K, v)$ generalized spin structure on an $S O(n)$ principal bundle $S O(n) \hookrightarrow P \rightarrow M$ is a cohomology class $\sigma \in H^{1}(P, K)$ whose restriction to the fiber is equal to $v$ in $H^{1}(S O(n), K) \cong \operatorname{Tor}_{2}(K)$. A $(K, v)$ generalized spin structure on an oriented manifold of dimension $\geq 3$ is a $(K, v)$ spin structure on its oriented framed bundle.

As usual this definition can be extended to dimensions less than 3 by using stabilization.

If $K$ is decomposed as a product of cyclic groups, $K=\mathbb{Z}_{d_{1}} \times \cdots \times \mathbb{Z}_{d_{k}}$, then a $(K, v)$ generalized spin structure is a sequence $\left(\sigma_{1}, \ldots, \sigma_{k}\right)$ where $\sigma_{j}$ is either a $d_{j}$-spin structure or a mod $d_{j}$ cohomology class, depending on the twist coefficient of the corresponding generator.

(a)

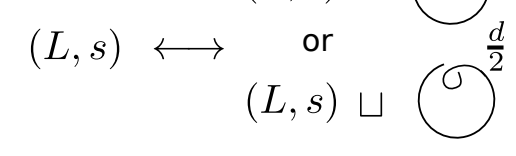

(b)

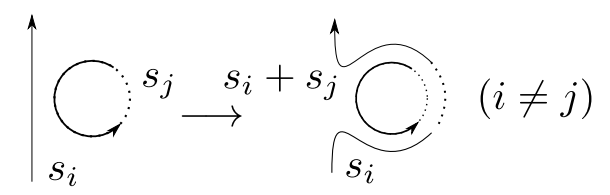

(c)

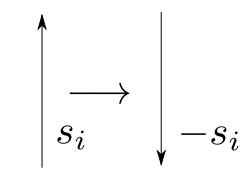

Figure 3.1. Refined $\operatorname{Spin}_{d}$-Kirby moves (a) Stabilization (b) Handle slide (c) Orientation reversal. Note that we use the blackboard framing and the labels refer to modulo $d$ characteristic solutions of $L$.

3.3. $\operatorname{Spin}_{d}$ Kirby moves. The second author proved the following result:

Theorem. [2] Let $(L, s)$ and $\left(L^{\prime}, s^{\prime}\right)$ be two oriented framed links with characteristic solutions $s, s^{\prime}$. Then, the manifolds $\left(S^{3}(L), s\right)$ and $\left(S^{3}\left(L^{\prime}\right), s^{\prime}\right)$ are $\mathrm{Spin}_{d}$-homeomorphic, if and only if, the pairs $(L, s)$ and $\left(L^{\prime}, s^{\prime}\right)$ are related by a finite sequence of the moves in Figure 3.1 or their inverses.

\section{From RibBon to MOdular CATEgories}

4.1. Ribbon categories. We introduce basic notions about ribbon categories following [20]. Without loss of generality, we work with monoidal categories that are strict (according to Mac Lane's coherence theorem [13], every monoidal category is equivalent to a strict one), i.e, categories with tensor product and unit object 1 satisfying

$$
\begin{array}{r}
V \otimes \mathbf{1}=\mathbf{1} \otimes V=V \\
(U \otimes V) \otimes W=U \otimes(V \otimes W) \\
f \otimes \mathrm{id}_{\mathbf{1}}=\mathrm{id}_{\mathbf{1}} \otimes f=f \\
(f \otimes g) \otimes h=f \otimes(g \otimes h)
\end{array}
$$


for any objects $U, V, W$ and morphisms $f, g, h$ of the category.

A braiding in a monoidal category $\mathcal{C}$ is a family of natural isomorphisms $b=\left\{b_{V, W}: V \otimes W \rightarrow\right.$ $W \otimes V\}$ where $V, W$ run over all the objects of $\mathcal{C}$, such that $b_{U, V \otimes W}=\left(\operatorname{id}_{V} \otimes b_{U, W}\right)\left(b_{U, V} \otimes \mathrm{id}_{W}\right)$ and $b_{U \otimes V, W}=\left(b_{U, W} \otimes \mathrm{id}_{V}\right)\left(\mathrm{id}_{U} \otimes b_{V, W}\right)$.

Let us consider the category $\left(\operatorname{Vect}_{\mathbf{k}}, \otimes\right)$ of finite dimensional vector spaces over a field $\mathbf{k}$. The family of maps $b_{V, W}: V \otimes W \rightarrow W \otimes V, b_{V, W}(v \otimes w)=w \otimes v$ for all $v \in V, w \in W$ defines a braiding.

A twist in a monoidal category $\mathcal{C}$ with braiding $b$ is a family of natural isomorphisms $\theta=$ $\left\{\theta_{V}: V \rightarrow V\right\}$ where $V$ runs over all the objects of $\mathcal{C}$, such that for any two objects $V, W$ of $\mathcal{C}$, we have $\theta_{V \otimes W}=b_{W, V} b_{V, W}\left(\theta_{V} \otimes \theta_{W}\right)$.

In the category $\left(\operatorname{Vect}_{\mathbf{k}}, \otimes, b\right)$, the family $\left\{\theta_{V}\right\}_{V}$ of maps $\theta_{V}: V \rightarrow V, \theta_{V}=\mathrm{id}_{V}$ defines a twist.

Let $\mathcal{C}$ be a monoidal category. Assume that to each object $V$ of $\mathcal{C}$ there are associated an object $V^{*}$ and two morphisms $c_{V}: \mathbf{1} \rightarrow V \otimes V^{*}, d_{V}: V^{*} \otimes V \rightarrow \mathbf{1}$. The rule $V \mapsto\left(V^{*}, c_{V}, d_{V}\right)$ is called duality in $\mathcal{C}$ if $\left(\operatorname{id}_{V} \otimes d_{V}\right)\left(c_{V} \otimes \mathrm{id}_{V}\right)=\mathrm{id}_{V}$ and $\left(d_{V} \otimes \operatorname{id}_{V^{*}}\right)\left(\operatorname{id}_{V^{*}} \otimes c_{V}\right)=\mathrm{id}_{V^{*}}$ for all objects $V$ of $\mathcal{C}$. The duality is called compatible with the braiding $b$ and the twist $\theta$ if in addition, for any object $V$ of $\mathcal{C}$ we have $\left(\theta_{V} \otimes \operatorname{id}_{V^{*}}\right) c_{V}=\left(\operatorname{id}_{V} \otimes \theta_{V^{*}}\right) c_{V}$.

For example, in $\left(\operatorname{Vect}_{\mathbf{k}}, \otimes\right)$, if we set $V^{*}:=\operatorname{Hom}_{\mathbf{k}}(V, \mathbf{k})$, fix a basis $\left\{e_{i}(V)\right\}_{i=1, \ldots, n}$ of $V$ with $n=\operatorname{dim}(V)$, and denote by $\left\{e_{i}(V)^{*}\right\}_{i=1, \ldots, n}$ its dual, we can define the map $c_{V}: \mathbf{k} \rightarrow V \otimes V^{*}$ by $c_{V}(1)=\sum_{i=1}^{n} e_{i}(V) \otimes e_{i}(V)^{*}$ and $d_{V}: V^{*} \otimes V \rightarrow \mathbf{k}$ by $d_{V}(f, v)=f(v)$ then, the rule $V \mapsto\left(V^{*}, c_{V}, d_{V}\right)$ defines a duality in $\operatorname{Vect}_{\mathbf{k}}$, compatible with the braiding $b$ and twist $\theta$ defined above.

Definition. A monoidal category $\mathcal{C}$ equipped with braiding $b$, twist $\theta$ and a compatible duality $(*, c, d)$ is called ribbon.

Example. (1) The category $\left(\operatorname{Vect}_{\mathbf{k}}, \otimes, b, \theta,(*, c, d)\right.$ ) defined above is ribbon;

(2) Let $\mathbf{U}_{q}(\mathfrak{g})$ be the quantum group corresponding to a semi-simple Lie algebra $\mathfrak{g}$, then the category of its finite dimensional representations $\operatorname{Rep}\left(\mathbf{U}_{q}(\mathfrak{g})\right)$ is ribbon [20].

In ribbon categories we can define traces of morphisms and dimensions of objects as follows.

Definition. (1) The quantum trace of an endomorphism $f: V \rightarrow V$ is the composition $\operatorname{tr}(f):=$ $d_{V} b_{V, V^{*}}\left(\left(\theta_{V} f\right) \otimes \operatorname{id}_{V^{*}}\right) c_{V}: \mathbf{1} \rightarrow \mathbf{1}$

(2) The quantum dimension of an object $V$ is $\langle V\rangle:=\operatorname{tr}\left(\mathrm{id}_{V}\right)$.

Proposition. In any ribbon category $\mathcal{C}$

(a) $\operatorname{tr}(f g)=\operatorname{tr}(g f)$ for any morphisms $f \in \operatorname{Hom}(V, W)$ and $g \in \operatorname{Hom}(W, V)$;

(b) $\operatorname{tr}(f \otimes g)=\operatorname{tr}(f) \operatorname{tr}(g)$ for any morphisms $f \in \operatorname{Hom}(V, W)$ and $g \in \operatorname{Hom}\left(V^{\prime}, W^{\prime}\right)$;

(c) $\langle V \otimes W\rangle=\langle V\rangle\langle W\rangle$ for any two objects $V, W$;

(d) $\langle V\rangle=\left\langle V^{*}\right\rangle$ for any object $V$.

4.1.1. The category of colored ribbon graphs. A ribbon graph is a compact, oriented surface in $\mathbb{R}^{2} \times[0,1]$ decomposed into bands, annuli and coupons. Bands are homeomorphic images of the square $[0,1] \times[0,1]$, annuli are homeomorphic images of the cylinder $S^{1} \times[0,1]$ and coupons are bands with a distinguished base, called bottom (the oposite base is called top). Isotopy of ribbon 
graphs means isotopy in the strip $\mathbb{R}^{2} \times[0,1]$, constant on the boundary intervals and preserving the decomposition into annuli, bands and coupons as well as preserving orientations.

Given a ribbon category $\mathcal{C}$, a ribbon graph is $\mathcal{C}$-colored if each band and each annulus is equipped with an object of $\mathcal{C}$, called color, and each coupon is colored by a morphism in $\mathcal{C}$. Isotopy of colored ribbon graphs means color-preserving isotopy. Starting with a ribbon category $\mathcal{C}$, one can construct the category $\operatorname{Rib}_{\mathcal{C}}$ of $\mathcal{C}$-colored ribbon graphs as follows: the objects are finite sequences of end points of ribbon graphs colored with objects of $\mathcal{C}$ or their duals, according to the orientation of strands, and the morphisms of $\mathrm{Rib}_{\mathcal{C}}$ are isotopy classes of $\mathcal{C}$-colored ribbon graphs (see Figure 4.1). The category Rib ${ }_{\mathcal{C}}$ can be given a tensor product, a natural braiding, twist and compatible duality and it becomes in this way ribbon.

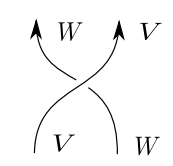

$X_{V, W}^{+}$
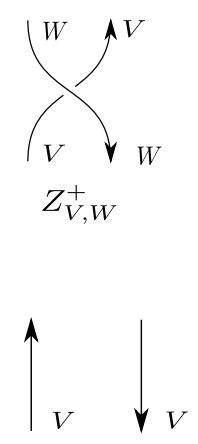

$i d_{V} \quad i d_{V^{*}}$

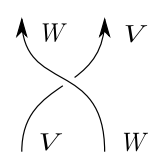

$X_{V, W}^{-}$

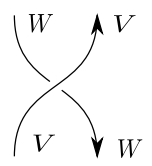

$Z_{V, W}^{-}$

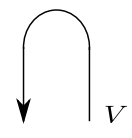

$\cap_{V}$

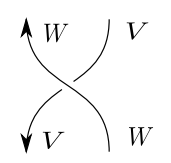

$Y_{V, W}^{+}$

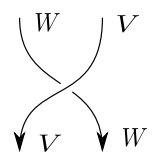

$T_{V, W}^{+}$

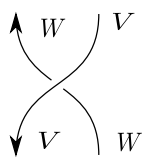

$Y_{V, W}^{-}$
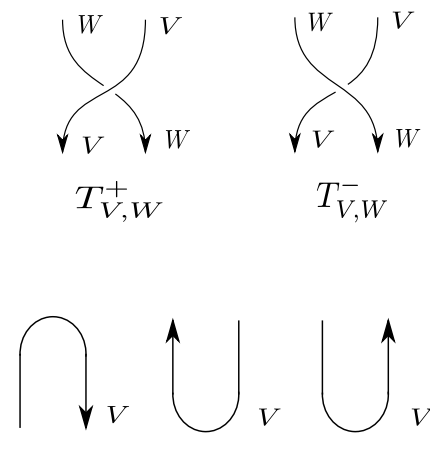

$\cap \bar{V}$

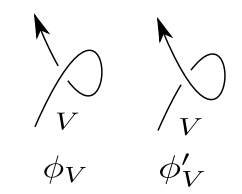

$\phi_{V} \quad \phi_{V}^{\prime}$

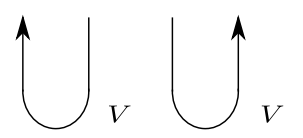

FiguRE 4.1. Morphisms in Rib

Ribbon categories turn out to play an important role in the theory of link invariants. The following theorem, due to Turaev, describes a way to associate to any ribbon category, invariants of colored ribbon graphs and in particular, of colored ribbon tangles (i.e. graphs without coupons).

Theorem ([20]). Let $\mathcal{C}$ be a ribbon category with a braiding $b$, a twist $\theta$ and a compatible duality $(*, c, d)$. Then there exists a unique covariant functor $F_{\mathcal{C}}: \mathrm{Rib}_{\mathcal{C}} \rightarrow \mathcal{C}$ preserving the tensor product such that (see Figure 4.1):

(1) $F_{\mathcal{C}}(V,+1)=V, F_{\mathcal{C}}(V,-1)=V^{*}$; 
(2) for any objects $V, W$ of $\mathcal{C}$, we have $F_{\mathcal{C}}\left(X_{V, W}^{+}\right)=b_{V, W} ; F_{\mathcal{C}}\left(\phi_{V}\right)=\theta_{V} ; F_{\mathcal{C}}\left(\cup_{V}\right)=c_{V} ; F_{\mathcal{C}}\left(\cap_{V}\right)=$ $d_{V}$

(3) for any elementary $\mathcal{C}$-colored ribbon graph (ribbon graph with one coupon) $\Gamma$, we have $F_{\mathcal{C}}(\Gamma)=f$ where $f$ is the color of the only coupon of $\Gamma$.

The functor $F_{\mathcal{C}}$ has the following properties:

$$
\begin{gathered}
F_{\mathcal{C}}\left(X_{V, W}^{-}\right)=\left(b_{W, V}\right)^{-1}, F_{\mathcal{C}}\left(Y_{V, W}^{+}\right)=\left(b_{W, V^{*}}\right)^{-1}, F_{\mathcal{C}}\left(Y_{V, W}^{-}\right)=b_{V^{*}, W} \\
F_{\mathcal{C}}\left(Z_{V, W}^{+}\right)=\left(b_{W^{*}, V}\right)^{-1}, F_{\mathcal{C}}\left(Z_{V, W}^{-}\right)=b_{V, W^{*}}, \\
F_{\mathcal{C}}\left(T_{V, W}^{+}\right)=b_{V^{*}, W^{*}}, F_{\mathcal{C}}\left(T_{V, W}^{-}\right)=\left(b_{W^{*}, V^{*}}\right)^{-1}, F_{\mathcal{C}}\left(\phi_{V}^{\prime}\right)=\theta_{V}^{-1} .
\end{gathered}
$$

In what follows we will use pictures to illustrate identities in a ribbon category. When drawing a pictorial identity, we always mean the corresponding morphisms in the ribbon category (see Figure 4.2 for an example).

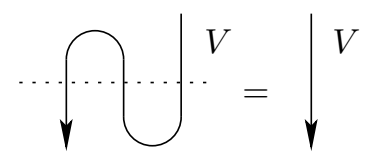

FiguRE 4.2. $\left(d_{V} \otimes \mathrm{id}_{V^{*}}\right)\left(\mathrm{id}_{V^{*}} \otimes c_{V}\right)=\mathrm{id}_{V^{*}}$

Corollary ([20]). Let $\Omega$ be a $\mathcal{C}$-colored ribbon graph that contains an annulus a. If $\Omega^{\prime}$ is the ribbon graph obtained from $\Omega$ by reversing the orientation of a and replacing the color of a with its dual object, then $F_{\mathcal{C}}\left(\Omega^{\prime}\right)=F_{\mathcal{C}}(\Omega)$.

4.2. Modular categories. Let $\mathbf{k}$ be a field of zero characteristic. A ribbon category is called $\mathbf{k}$-linear or pre-additive if the Hom sets are $\mathbf{k}$-vector spaces, composition and tensor product are bilinear and $\operatorname{End}(\mathbf{1})=\mathbf{k}$. An object $\lambda$ of the category is called simple if the map $u \mapsto u \cdot \mathrm{id}_{\lambda}$ from $\mathbf{k}=\operatorname{End}(\mathbf{1})$ to $\operatorname{End}(\lambda)$ is an isomorphism.

We will denote by $\mathcal{C}^{\oplus}$ the additive closure of a pre-additive ribbon category $\mathcal{C}$, which admits direct sums of objects and compositions of morphisms are modelled on the matrix multiplication ([13]).

Definition. A modular category over the field $\mathbf{k}$ is a $\mathbf{k}$-linear ribbon category $\mathcal{C}$ with a finite set of simple objects $\Gamma$ that satifies the following axioms:

(1) Normalization: the trivial object $\mathbf{1}$ is in $\Gamma$;

(2) Duality: for any object $\lambda \in \Gamma$, its dual $\lambda^{*}$ is isomorphic to an object in $\Gamma$;

(3) Domination: for any object $V$ of the category, there exists a finite decomposition

$$
\operatorname{id}_{V}=\sum_{i} f_{i} \operatorname{id}_{\lambda_{i}} g_{i}
$$

with $\lambda_{i} \in \Gamma, \forall i$; 


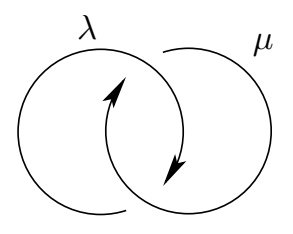

Figure 4.3. The Hopf link with linking number +1 and colors $\lambda$ and $\mu$

(4) Non-degeneracy: the matrix $S=\left(S_{\lambda \mu}\right)_{\lambda, \mu \in \Gamma}$ is invertible over $\mathbf{k}$, where $S_{\lambda \mu} \in \mathbf{k}$ is the endomorphism of the trivial object associated with the $(\lambda, \mu)$-colored, 0-framed Hopf link with linking +1 .

The category is called pre-modular if we remove the last axiom. Replacing $\mathcal{C}$ with its additive closure, we can reformulate the domination axiom as follows: for any $\lambda, \nu, \mu \in \Gamma$ there exist positive integers $C_{\lambda \nu}^{\mu}$ called structure constants, such that $\lambda \otimes \nu \simeq \oplus_{\mu \in \Gamma} C_{\lambda \nu}^{\mu} \mu$. The domination axiom says that any object decomposes into a direct sum of simple ones.

Example. Let $\mathfrak{g}=\mathfrak{s l}_{N}$ and $q$ be an $(N+K)$ th root of unity. Then, there exists an associated modular category $\mathcal{C}(N, K)(N, K \geq 2)$ with simple objects given by partitions $\lambda$ from

$$
\Gamma=\left\{\lambda=\left(\lambda_{1}, \ldots, \lambda_{s}\right) \mid \lambda_{1} \leq K, s<N\right\} .
$$

See e.g. [3] for more details.

4.3. Properties of pre-modular categories. In what follows, we describe basic properties of pre-modular categories that will be relevant to the rest of the paper. We follow the lines of [1]. Unless otherwise stated, $\mathcal{C}$ is a pre-modular category over a field $\mathbf{k}$ with zero characteristic and $\Gamma$ is the set of representatives of simple objects.

Definition. (a) An object $\lambda$ of $\mathcal{C}$ is called transparent if for any object $\nu$ the following morphisms in $\mathcal{C}$ are equal

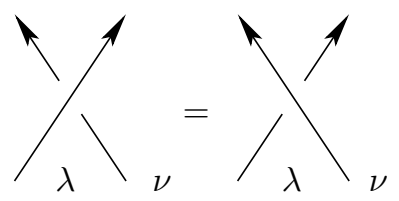

(b) A morphism $f \in \operatorname{Hom}(\lambda, \nu)$ is called negligible if $\operatorname{tr}(f g)=0$ for any $g \in \operatorname{Hom}(\nu, \lambda)$.

(c) The braiding coefficients between two objects $\lambda, \nu \in \Gamma$ are defined as a collection $\left\{b_{\lambda, \nu}^{\mu}\right\}$ for all $\mu \subset \lambda \otimes \nu$ such that

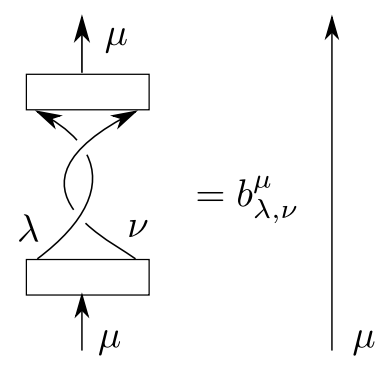




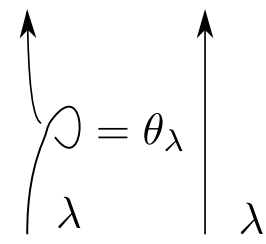

Figure 4.4. Twist coefficient

(d) For any object $\lambda \in \Gamma$ the twist coefficient $\theta_{\lambda}$ is defined by the equality:

We discuss in detail a formula that will be used extensively throughout the paper. Let us start by fixing some notations. Given objects $\alpha_{1}, \cdots, \alpha_{n}, \beta_{1}, \cdots, \beta_{m} \in \Gamma$, we denote by $H_{\alpha_{1}}^{\beta_{1}, \cdots, \alpha_{n}}$ the $\mathbf{k}$-module of morphisms $\operatorname{Hom}_{\mathcal{C}}\left(\alpha_{1} \otimes \cdots \otimes \alpha_{n}, \beta_{1} \otimes \cdots \otimes \beta_{m}\right)$.

The modules $H_{\mu}^{\lambda \nu}, H_{\mu \nu^{*}}^{\lambda}, H_{\nu^{*}}^{\mu^{*} \lambda}, H_{\nu^{*} \lambda^{*}}^{\mu^{*}}, H_{\lambda^{*}}^{\nu \mu^{*}}$ and $H_{\lambda^{*} \mu}^{\nu}$ are mutually isomorphic, as well as the modules $H_{\mu \nu^{*} \lambda^{*}}, H^{\lambda \nu \mu^{*}}$ and all obtained from them by a cyclic permutation of colors. Identifying these modules along the isomorphisms, we get a symmetrized multiplicity module $\tilde{H}^{\lambda \nu \mu^{*}}$ for which only the cyclic order of colors is important. The elements of $\tilde{H}^{\lambda \nu \mu^{*}}$ are represented by a round coupon with one incoming line (colored with $\mu$ ) and two outgoing ones (colored by $\lambda$ and $\nu$ ).

The pairing $\langle\rangle:, \tilde{H}^{\lambda \nu \mu^{*}} \times \tilde{H}^{\mu \lambda^{*} \nu^{*}} \rightarrow \mathbf{k}$ defined as $\langle f, g\rangle=\operatorname{tr}(f g)$ is non-degenerate since the category $\mathcal{C}$ can be assumed without negligible morphisms (if any, they can be quotiened out). The symmetrized modules $\tilde{H}^{\lambda \nu \mu^{*}}$ and $\tilde{H}^{\mu \lambda^{*} \nu^{*}}$ are dual to each other, therefore we can choose bases $\left(a_{i}\right)_{i \in I_{\lambda \nu}^{\mu}}$ for $\tilde{H}^{\mu \lambda^{*} \nu^{*}}$ and $\left(b_{i}\right)_{i \in I_{\lambda \nu}^{\mu}}$ for the module $\tilde{H}^{\lambda \nu \mu^{*}}$ that are dual with respect to $\langle$,$\rangle .$ Note that the composition $b_{j} \circ a_{i}$ is an endomorphism of the simple object $\mu$, so it is of the form $c_{i j} \cdot \mathrm{id}_{\mu}, c_{i j} \in \mathbf{k}$. Comparing the traces gives $\delta_{i j}=c_{i j}\langle\mu\rangle$ so $b_{j} \circ a_{i}=\delta_{i j}\langle\mu\rangle^{-1} \mathrm{id}_{\mu}$. For any simple objects $\lambda, \nu \in \Gamma$, the domination axiom applied to $\mathrm{id}_{\lambda \otimes \nu}$ yields the following relation, known as the fusion formula:

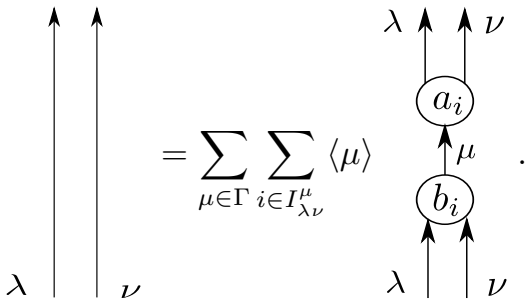

For any pre-modular category $\mathcal{C}$, let us define the Kirby color $\omega=\sum_{\lambda \in \Gamma}\langle\lambda\rangle \lambda$ as an element of $K_{0}\left(\mathcal{C}^{\oplus}\right) \otimes \mathbf{k}$, where $K_{0}\left(\mathcal{C}^{\oplus}\right)$ denotes the Grothendieck ring of the category $\mathcal{C}^{\oplus}$.

Proposition (Sliding property, [1]). In any pre-modular category the following handle sliding identity holds

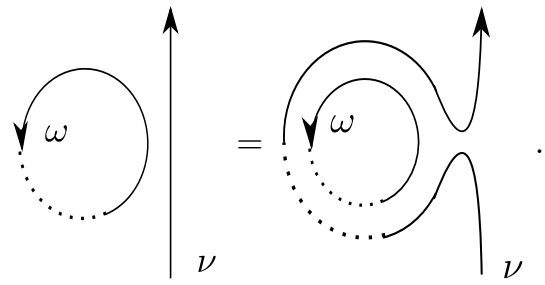


Lemma (Killing property, [1]). Let $\mathcal{C}$ be a pre-modular category with $\langle\omega\rangle \neq 0$ and $\lambda \in \Gamma$. Then, the morphism

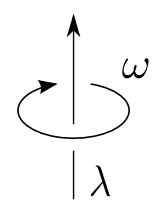

is non-trivial if and only if $\lambda$ is transparent.

Proof. If $\lambda$ is transparent, then the map

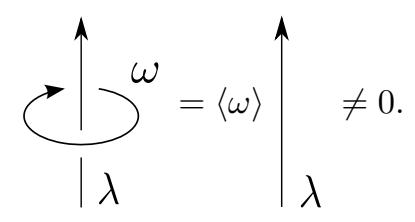

Conversely, if we assume that for some $c \in \mathbf{k}, c \neq 0$ we have

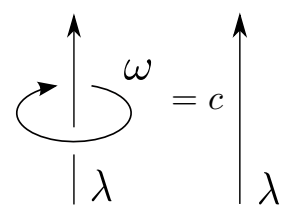

then, by sliding any $\nu$-colored strand along the $\omega$-colored one, we obtain the following equalities of morphisms in $\mathcal{C}$

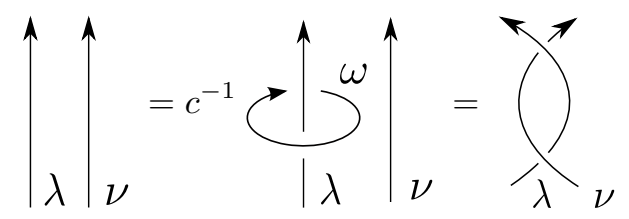

and therefore, $\lambda$ is transparent.

Proposition ([1]). A pre-modular category with $\langle\omega\rangle \neq 0$ and with no non-trivial transparent objects is modular.

Proof. Let $\bar{S}=\left(\bar{S}_{\lambda \nu}\right)_{\lambda \nu}$ be a matrix with entries given by the relation

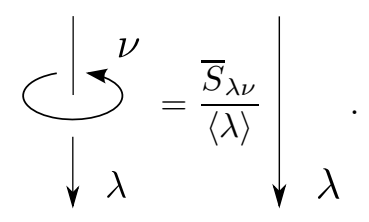


We want to prove that the product $S \cdot \bar{S}=\langle\omega\rangle \mathrm{I}$, where I is the identity matrix of size $|\Gamma|$. We have

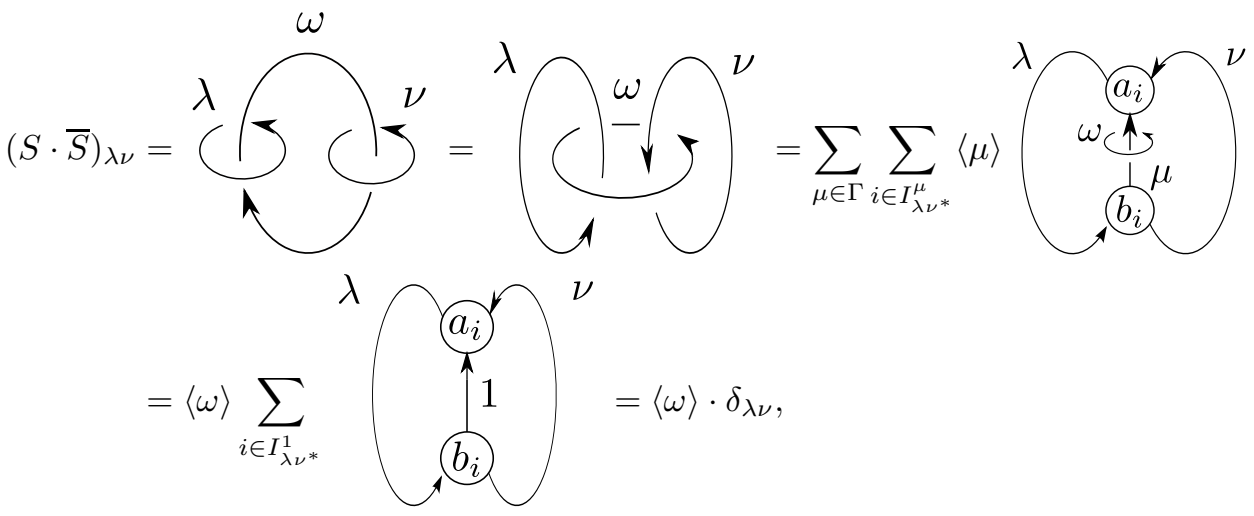

where $\delta_{\lambda \nu}$ is the Kronecker index. The second equality holds by isotopy, the third equality holds by the fusion formula, while the fourth equality is a consequence of the killing property. The last equality can be proved using the structure of the modules $\operatorname{Hom}\left(\mathbf{1}, \lambda \otimes \nu^{*}\right)$ and $\operatorname{Hom}\left(\lambda \otimes \nu^{*}, \mathbf{1}\right)$.

Note. If $\mathcal{C}$ is a modular category, then $\langle\omega\rangle$ is invertible in $\mathbf{k}$, and hence $\langle\omega\rangle \neq 0$.

4.4. WRT invariants. Given a modular category $\mathcal{C}$ and a closed 3-manifold $M=S^{3}(L)$ obtained by surgery on $S^{3}$ along a framed link $L$, whose linking matrix has $b_{+}$positive and $b_{-}$ negative eigenvalues, we define

$$
\tau_{\mathcal{C}}(M)=\frac{F_{\mathcal{C}}(L(\omega, \ldots, \omega))}{F_{\mathcal{C}}\left(U_{1}(\omega)\right)^{b_{+}} F_{\mathcal{C}}\left(U_{-1}(\omega)\right)^{b_{-}}}
$$

where $U_{ \pm 1}$ denotes the \pm 1 -framed unknot and $F_{\mathcal{C}}: \operatorname{Rib}_{\mathcal{C}} \rightarrow \mathcal{C}$ is the natural ribbon functor.

Theorem ([20]). For any modular category $\mathcal{C}, \tau_{\mathcal{C}}(M)$ defines a topological invariant of $M$, independent on the choice of the link $L$.

Proof. We need to show that $\tau_{\mathcal{C}}(M)$ is well-defined and does not change under Kirby moves.

The fact that $F_{\mathcal{C}}\left(U_{ \pm 1}(\omega)\right)$ are non-zero follows from the properties of the ribbon functor $F_{\mathcal{C}}$ and the non-degeneracy axiom for $\mathcal{C}$.

The first Kirby move is easy to establish. The invariance under the second Kirby move is provided by Proposition 4.3. In order to prove invariance under orientation reversal, let us consider the link $L^{\prime}$ obtained from $L$ by reversing the orientation of a component $L_{k}$. Without loss of generality, we may assume $k=1$. Thanks to Corollary 4.1.1 we have

$$
\begin{aligned}
F_{\mathcal{C}}\left(L^{\prime}(\omega, \cdots, \omega)\right) & =\sum_{\lambda \in \Gamma}\langle\lambda\rangle F_{\mathcal{C}}\left(L^{\prime}(\lambda, \omega, \cdots, \omega)\right)=\sum_{\lambda \in \Gamma}\left\langle\lambda^{*}\right\rangle F_{\mathcal{C}}\left(L\left(\lambda^{*}, \omega, \cdots, \omega\right)\right) \\
& =\sum_{\mu \in \Gamma}\langle\mu\rangle F_{\mathcal{C}}(L(\mu, \omega, \cdots, \omega))=F_{\mathcal{C}}(L(\omega, \cdots, \omega)) .
\end{aligned}
$$

The linking matrix $\left(L_{i j}^{\prime}\right)={ }^{t} S\left(L_{i j}\right) S$ with $S=\operatorname{diag}(-1,1, \cdots, 1)$ hence the matrices $\left(L_{i j}^{\prime}\right)$ and $\left(L_{i j}\right)$ have the same eigenvalues. In particular, $b_{+}^{\prime}=b_{+}, b_{-}^{\prime}=b_{-}$so $\tau_{\mathcal{C}}\left(M, L^{\prime}\right)=\tau_{C}(M, L)$. This concludes the proof. 


\section{Group-CATEgories From inVERTible obJects}

In this section, we show that for any modular category $\mathcal{C}$, its group of invertible objects $G$ defines the structure of $\widehat{G}$-category on $\mathcal{C}$. We identify an object with its isomorphism class and hence equality between objects means an isomorphism.

5.1. Invertible objects. An object $\lambda$ in $\Gamma$ is called invertible if there exists another object $\nu \in \Gamma$ such that $\lambda \otimes \nu=1$. Isomorphism classes of invertible simple objects form a finite abelian group under tensor multiplication. Let us denote this group by $G \subset \Gamma$ and let $\widehat{G}=\operatorname{Hom}\left(G, \mathbb{C}^{*}\right)$ be the group of its characters. We say that the tensor order of $\lambda$ is $d$ if $\lambda^{\otimes d}=1$ with $d$ minimal.

Lemma. For any $\lambda \in \Gamma$ and any invertible $g \in G, \lambda \otimes g \in \Gamma$.

Proof. By the domination axiom we have $\lambda \otimes g=\oplus_{i} \mu_{i}$. Multiplying this identity by the inverse of $g$, we get $\lambda=\oplus_{i}\left(\mu_{i} \otimes g^{-1}\right)$. Thus, the right hand is simple, and so $\lambda \otimes g$ has to be simple too.

Example. In Example 4.2 the object $K$ (the longest row) is invertible of order $N$.

5.2. Group-category. Assume $\mathcal{C}$ is a modular category with $G$ the group of invertible simple objects. We can define a $\widehat{G}$-structure on $\mathcal{C}$ as follows: Given $\lambda \in \Gamma$, the braiding coefficient of $\lambda$ with elements of $G$ defines a map $\Gamma \rightarrow \widehat{G}$ which associates to each $\lambda$ a character $\chi_{\lambda} \in \widehat{G}$ defined by the equality:

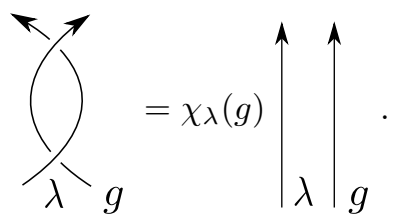

Indeed, the previous lemma implies that the braiding operator acts on $\lambda$ and $g$ as a multiplication by $S_{\lambda g} /\langle\lambda\rangle\langle g\rangle$, i.e. only one braiding coefficient is non-zero. Using the fact that $g$ is of finite order, we deduce that this coefficient is a root of unity of that order. Observe that $\chi_{\lambda \otimes \mu}=\chi_{\lambda} \chi_{\mu}$ defines a group multiplication on $\widehat{G}$. By taking a logarithm of the character, we can identify $G$ with its dual $\widehat{G}$ and write the group operation on $G$ additively.

Clearly, $\mathcal{C}$ splits into a disjoint union of subcategories $\mathcal{C}_{\alpha}$ for $\alpha \in \widehat{G} \cong G$. Moreover, for any $\lambda, \mu \in \Gamma, \operatorname{Hom}(\lambda, \mu)$ is either zero if $\lambda \neq \mu$ or $\mathbf{k}$ otherwise (by the assumption these objects are simple). Hence, we just proved the following.

Proposition. A modular category $\mathcal{C}$ with a group $G$ of invertible objects splits as a disjoint union of subcategories $\left\{\mathcal{C}_{\alpha}\right\}_{\alpha \in G}$ such that

- each $\mathcal{C}_{\alpha}$ is a full subcategory of $\mathcal{C}$;

- each object of $\mathcal{C}$ belongs to $\mathcal{C}_{\alpha}$ for a unique $\alpha$;

- $\operatorname{Hom}_{\mathcal{C}}\left(\mathcal{C}_{\alpha}, \mathcal{C}_{\beta}\right)=0$ if $\alpha \neq \beta$;

- for $U \in \mathcal{C}_{\alpha}$ and $V \in \mathcal{C}_{\beta}, U \otimes V \in \mathcal{C}_{\alpha+\beta}$;

- $1 \in \mathcal{C}_{0}$, and for $U \in \mathcal{C}_{\alpha}, U^{*} \in \mathcal{C}_{-\alpha}$.

Note that this is a special case of group-categories defined by Turaev in [21].

If $\lambda \in \mathcal{C}_{\alpha}$, then we will call $\alpha$ degree of $\lambda$ and denote by by $\operatorname{deg}(\lambda)$. 
If $G$ is cyclic of order $d$, we call $\mathcal{C}$ a modular $d$-category. Fixing a generator $t$ of $G$ and a primitive $d$ th root of unity $e_{d}$, we have the decomposition

$$
\Gamma=\Gamma_{0} \amalg \Gamma_{1} \amalg \cdots \amalg \Gamma_{d-1},
$$

where $\Gamma_{i}=\left\{\lambda \in \Gamma \mid \chi_{\lambda}(t)=e_{d}^{i}\right\}$.

Example. Let us assume that $N=(N, K)$ in Examples 4.2 and 5.1. Then $G=\mathbb{Z}_{N}$ is generated by $K$. Hence, the category $\mathcal{C}(N, K)$ is a modular $N$-category with $\operatorname{deg}(\lambda)=\sum_{i} \lambda_{i}(\bmod N)$.

We will need the following lemma.

Lemma. Let $\mathcal{C}$ be a modular $d$-category such that the group $G=\langle t\rangle \cong \mathbb{Z}_{d}$. Then $\langle t\rangle=1$ if $d$ is odd, and $\langle t\rangle= \pm 1$ if $d$ is even.

Proof. Let $b_{t, t}$ be the braiding coefficient, such that

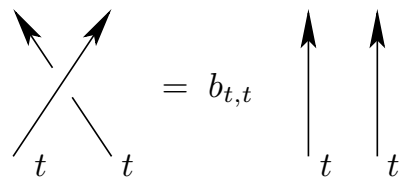

Using

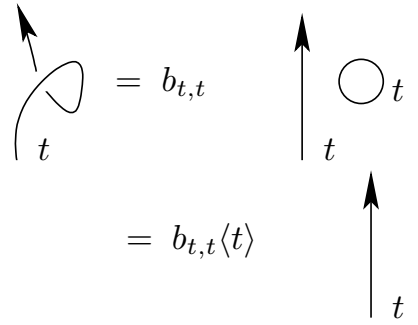

we have $\theta_{t}=b_{t, t}\langle t\rangle$. On the other hand, from Definition 4.1 we get $\theta_{t} b_{t, t^{*}}=1$, where $t^{*}=t^{d-1}$, so $\langle t\rangle\left(b_{t, t}\right)^{d}=1$. Since $\left(b_{t, t}\right)^{2 d}=1$, it follows that $\langle t\rangle= \pm 1$. If $d$ id odd, then $\left\langle t^{\otimes d}\right\rangle=\langle 1\rangle=1=$ $\langle t\rangle$.

Let us recall the following definition which will play a central role in our exposition.

Definition. Let $H \subset G$ be a subgroup. A modular category with a group $G$ of invertible objects is called $H$-refinable if $H \subset \mathcal{C}_{0}$. If, in addition, $H$ has at least one element with twist coefficient -1 , we call the category $H-$ spin.

In the case $H$ is cyclic of order $d$, we will use the shorthand $d$-refinable and $d$-spin.

Example. Given an even integer $d=(N, K)=\alpha \beta$, such that $\left(\alpha, N^{\prime}\right)=\left(\beta, K^{\prime}\right)=1$ for $N^{\prime}=\frac{N}{d}$ and $K^{\prime}=\frac{K}{d}$, The second author constructed in [3] a category $\dot{\mathcal{C}}(N, K)$ with

$$
\dot{\Gamma}=\left\{\left(1^{N}\right)^{\otimes i} \otimes \lambda \mid 0 \leq i<\alpha, \lambda_{1} \leq K, \lambda_{1}^{\vee}<N\right\}
$$

and $G=\mathbb{Z}_{d}$ generated by $1^{N} \otimes K$. Here $\lambda^{\vee}$ denotes the transpose partition. The second author proved that the category $\dot{\mathcal{C}}(N, K)$ is $d$-spin modular. 
5.3. Sliding identities in pre-modular group-categories. Assume $\mathcal{C}$ is a pre-modular $d-$ category. For any $0 \leq u<d$, let us define the refined Kirby colors $\omega_{u}=\sum_{\lambda \in \Gamma_{u}}\langle\lambda\rangle \lambda$ as objects in the additive closure of $\mathcal{C}$.

Lemma (Graded sliding property). In any pre-modular $d$-category $\mathcal{C}$ we have the following equality of morphisms:

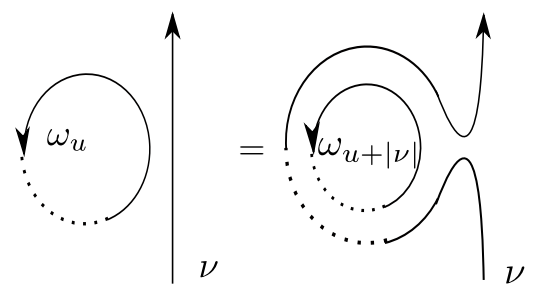

with $|\nu|=\operatorname{deg}(\nu)$.

Proof. The proof is the same as in the non-graded case, using the fact that $\operatorname{Hom}(\lambda \otimes \nu, \mu)$ is zero unless $\operatorname{deg}(\mu)=\operatorname{deg}(\lambda)+\operatorname{deg}(\nu)$. Hence, the sum over $\mu$ can be restricted to $\Gamma_{u+|\nu|}$.

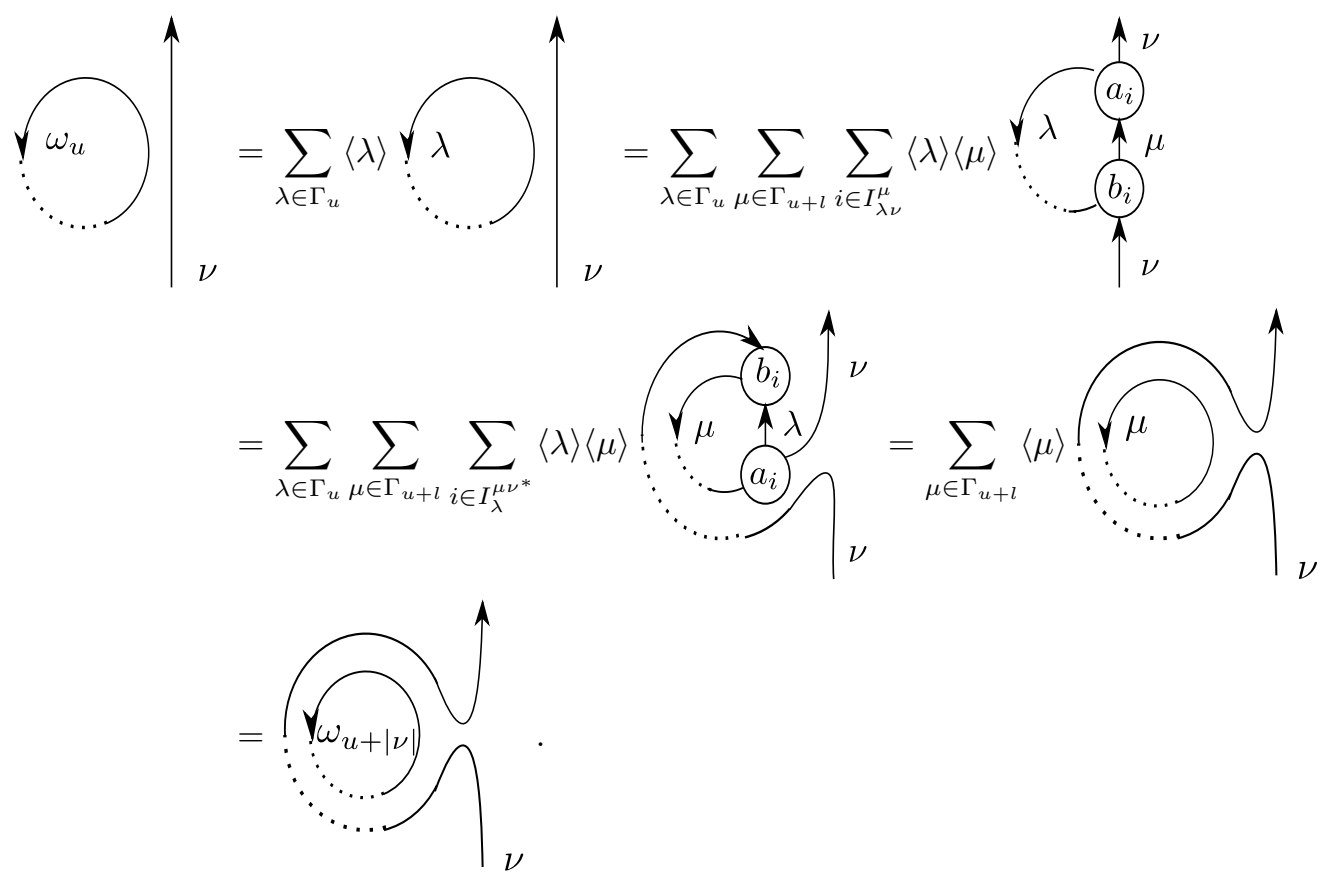

In the second and the fourth equalities we use the fusion formula, the third equality holds by isotopy.

For any $0 \leq v<d$, and a primitive $d$ th root of unity $e_{d}$, we define

$$
\omega^{v}=\sum_{\lambda \in \Gamma} e_{d}^{v \operatorname{deg}(\lambda)}\langle\lambda\rangle \lambda
$$

the dual refined Kirby color. Note that $\omega^{0}=\omega$, however other dual Kirby colors depend on the choice of $e_{d}$. We use the graded sliding identity to prove the following lemma. 
Proposition (Dual sliding property). In any pre-modular $d$-category $\mathcal{C}$ we have the following equality of morphisms:

(a)

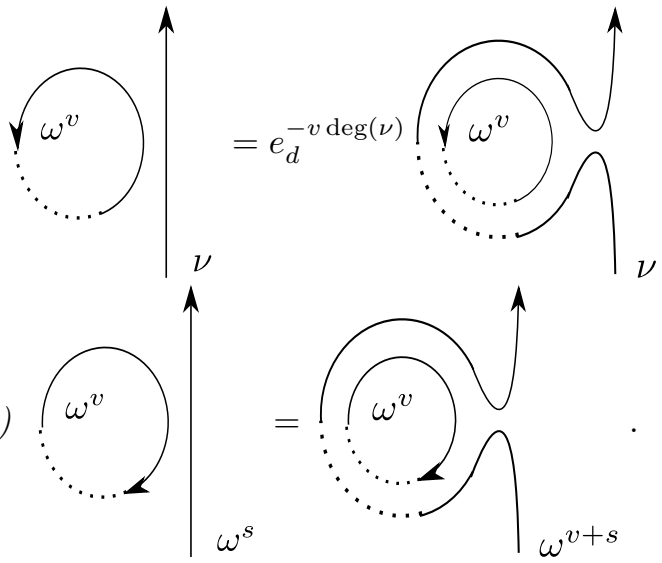

Proof. (a) We have the following equalities:

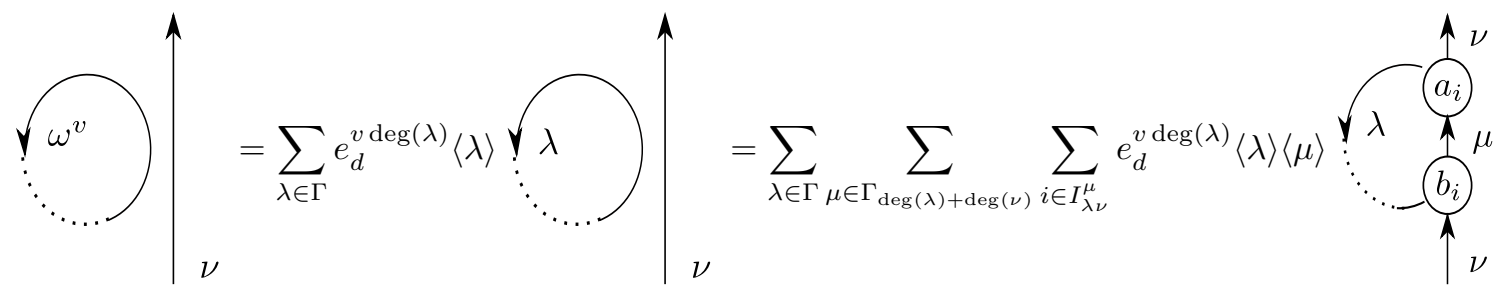

$$
\left.=e_{d}^{-v \operatorname{deg}(\nu)} \sum_{\mu \in \Gamma} \sum_{\lambda \in \Gamma_{\operatorname{deg}(\mu)-\operatorname{deg}(\nu)}} e_{d}^{v \operatorname{deg}(\mu)}\langle\lambda\rangle\langle\mu\rangle\right\rangle
$$

The second and fourth equalities follow from the fusion formula, the third equality holds by isotopy.

(b) According to Corollary 4.1.1, given a $\mathcal{C}$-colored ribbon graph $\Omega$ with an annulus component $a$ colored with the dual Kirby color $\omega^{s}$, if we consider the ribbon graph $\Omega^{\prime}$ obtained from $\Omega$ by reversing the orientation of $a$ and changing its color to $\omega^{-s}$, then $F_{\mathcal{C}}(\Omega)=F_{\mathcal{C}}\left(\Omega^{\prime}\right)$. In particular, 
we have
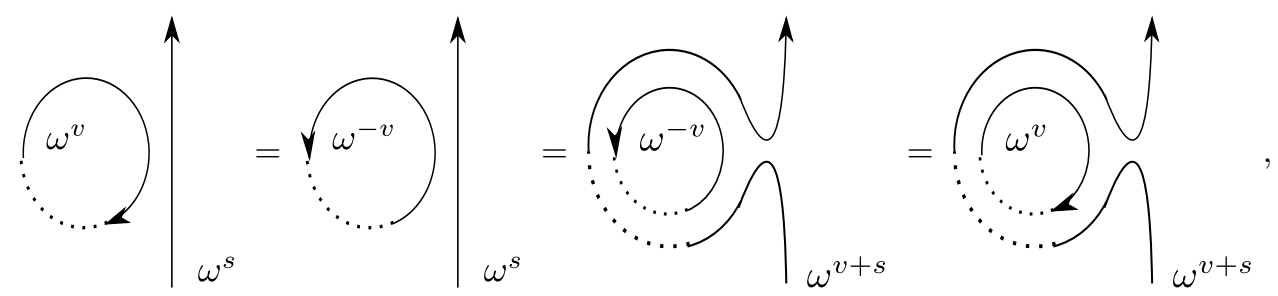

where the second equality is a straightforward application of (a).

\section{Spin modular CATEgories}

This section is devoted to the proof of Theorem 1. Motivated by the known examples we will first give the proof in the cyclic case. For $d$-spin modular categories we obtain invariants of 3 -manifolds equipped with $\operatorname{Spin}_{d}$ structure. In cohomological case we get refined invariants for 3 -manifolds with modulo $d$-dimensional cohomology classes.

For the rest of this section $M=S^{3}(L)$ is a closed 3-manifold obtained by surgery on $S^{3}$ along a framed link $L=\left(L_{1}, \cdots, L_{n}\right)$ whose linking matrix $\left(L_{i j}\right)$ has $b_{+}$positive and $b_{-}$negative eigenvalues.

6.1. Spin refinements, cyclic case. Let $d$ be an even, positive integer and $\mathcal{C}$ be a $d$-spin modular category. For any $s=\left(s_{1}, \ldots, s_{n}\right) \in \operatorname{Spin}_{d}(M)$ let us define

$$
\tau_{\mathcal{C}}(M, s)=\frac{F_{\mathcal{C}}\left(L\left(\omega_{s_{1}}, \ldots, \omega_{s_{n}}\right)\right)}{F_{\mathcal{C}}\left(U_{1}(\omega)\right)^{b}+F_{\mathcal{C}}\left(U_{-1}(\omega)\right)^{b-}} .
$$

Theorem. For any $d$-spin modular category $\mathcal{C}, \tau_{\mathcal{C}}(M, s)$ is a topological invariant of the pair $(M, s)$. Moreover,

$$
\tau_{\mathcal{C}}(M)=\sum_{s \in \operatorname{Spin}_{d}(M)} \tau_{\mathcal{C}}(M, s) .
$$

Proof. To prove the first statement we need to show that $\tau(M, s)$ is well-defined and does not change under refined Kirby moves.

Invariance under the first two $\operatorname{Spin}_{d}$ Kirby moves follows immediately from the graded sliding property and the next lemma which implies that $F_{\mathcal{C}}\left(U_{\epsilon}(w)\right)=F_{\mathcal{C}}\left(U_{\epsilon}\left(w_{\frac{d}{2}}\right)\right)$ is invertible for $\epsilon= \pm 1$. In order to prove invariance under orientation reversal, let us consider the link $L^{\prime}$ obtained from $L$ by reversing the orientation of a component $L_{k}$. Without loss of generality, we may assume that $k=1$. We have seen in the proof of Theorem 4.4 that the linking matrices $\left(L_{i j}^{\prime}\right)$ and $\left(L_{i j}\right)$ have the same eigenvalues so $b_{+}^{\prime}=b_{+}, b_{-}^{\prime}=b_{-}$.

Since $s^{\prime}=\left(-s_{1}, s_{2}, \cdots, s_{n}\right)$ applying Corollary 4.1 .1 gives

$$
\begin{aligned}
F_{\mathcal{C}}\left(L^{\prime}\left(\omega_{-s_{1}}, \omega_{s_{2}}, \cdots, \omega_{s_{n}}\right)\right) & =\sum_{\lambda \in \Gamma_{-s_{1}}}\langle\lambda\rangle F_{\mathcal{C}}\left(L^{\prime}\left(\lambda, \omega_{s_{2}}, \cdots, \omega_{s_{n}}\right)\right) \\
& =\sum_{\lambda \in \Gamma_{-s_{1}}}\left\langle\lambda^{*}\right\rangle F_{\mathcal{C}}\left(L\left(\lambda^{*}, \omega_{s_{2}}, \cdots, \omega_{s_{n}}\right)\right) \\
& =\sum_{\mu \in \Gamma_{s_{1}}}\langle\mu\rangle F_{\mathcal{C}}\left(L\left(\mu, \omega_{s_{2}}, \cdots, \omega_{s_{n}}\right)\right) \\
& =F_{\mathcal{C}}\left(L\left(\omega_{s_{1}}, \omega_{s_{2}}, \cdots, \omega_{s_{n}}\right)\right)
\end{aligned}
$$


and therefore $\tau_{\mathcal{C}}\left(M, L^{\prime} ; s^{\prime}\right)=\tau_{\mathcal{C}}(M, L ; s)$. It remains to prove that $F_{\mathcal{C}}\left(L\left(\omega_{s_{1}}, \ldots, \omega_{s_{n}}\right)\right)=0$ if $s_{i}$ do not solve $\sum_{j=1}^{n} L_{i j} s_{j}=\frac{d}{2} L_{i i}(\bmod d)$. The proof is in three steps.

Assume that the first component $L_{1}$ of our link is the \pm 1 -framed unknot. Then it can be unlinked from the rest of $L$ by applying the Fenn-Rourke move. The graded sliding identity and Lemma 6.1 tell us that $s_{1}$ should solve the above equations.

Assume $L_{1}$ is the $a$-framed unknot. Then we add a \pm 1 -framed unknot to our link (with an invertible invariant) and slide it along $L_{1}$ (perform the inverse Fenn-Rourke move). This changes the framing on $L_{1}$ by $\mp 1$ and allows to reduce this case to the previous one.

Finally, assume $L_{1}$ is arbitrary. Then we can unknot $L_{1}$ by adding \pm 1 -framed unknots to our link in such a way, that their linking number with $L_{1}$ is zero. This again reduces the situation to the previous case.

Lemma. For any $d$-spin modular category $\mathcal{C}, F_{\mathcal{C}}\left(U_{ \pm 1}\left(w_{u}\right)\right)$ is zero unless $u=\frac{d}{2}$.

Proof. Recall that invertible objects form an abelian group under tensor multiplication, which acts on $\Gamma$. In particular, its cyclic subgroup $H=\langle t\rangle \cong \mathbb{Z}_{d}$ acts on each $\Gamma_{u}$. Let us denote by $\widetilde{\Gamma}_{u}$ the set of orbits under this action and by $\widetilde{\omega}_{u}$ the corresponding reduced Kirby color. Note that $\omega_{u}=\sum_{i=0}^{d-1}\langle t\rangle^{i} t^{i} \widetilde{\omega}_{u}$. Let $H_{a, b}$ be the $(a, b)$-framed Hopf link with linking matrix -1 .

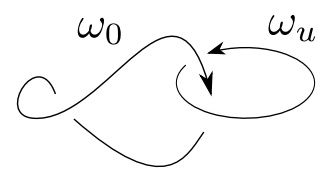

Figure 6.1. The Hopf link $H_{1,0}\left(\omega_{0}, \omega_{u}\right)$.

After sliding the second component of $H_{(1,0)}\left(\omega_{0}, \omega_{u}\right)$ along the first one we get

$$
\begin{aligned}
F_{\mathcal{C}}\left(U_{1}\left(w_{u}\right)\right) F_{\mathcal{C}}\left(U_{-1}\left(w_{u}\right)\right) & =F_{\mathcal{C}}\left(H_{1,0}\left(\omega_{0}, \omega_{u}\right)\right) \\
& =\sum_{i, j=0}^{d-1}\langle t\rangle^{i+j} F_{\mathcal{C}}\left(H_{1,0}\left(t^{i} \widetilde{\omega}_{0}, t^{j} \widetilde{\omega}_{u}\right)\right) \\
& =\sum_{i, j=0}^{d-1}\langle t\rangle^{i+j} F_{\mathcal{C}}\left(H_{1,0}\left(\widetilde{\omega}_{0}, \widetilde{\omega}_{u}\right)\right) e_{d}^{u i} \theta_{t}^{i}\langle t\rangle^{i+j} \\
& =d F_{\mathcal{C}}\left(H_{1,0}\left(\widetilde{\omega}_{0}, \widetilde{\omega}_{u}\right)\right) \sum_{i=0}^{d-1} e_{d}^{u i}(-1)^{i}
\end{aligned}
$$

which is zero unless $u=\frac{d}{2}(\bmod d)$. Here we used that $\theta_{t}=-1$ and $\langle t\rangle= \pm 1$, by Lemma 5.2 . Since $F_{\mathcal{C}}\left(U_{1}\left(w_{u}\right)\right)$ and $F_{\mathcal{C}}\left(U_{-1}\left(w_{u}\right)\right)$ are complex conjugate to each other, the result follows.

6.2. Cohomological refinements. In this subsection we assume that $\mathcal{C}$ is a non-spin $d$-refinable modular category. 
The elements $h \in H^{1}\left(M ; \mathbb{Z}_{d}\right)$ are combinatorially given by solutions of $\sum_{j=1}^{n} L_{i j} h_{j}=0$ $(\bmod d), \forall i=1, \ldots, n$. Let $L$ be an oriented framed link and $h \in H^{1}\left(S^{3}(L) ; \mathbb{Z}_{d}\right)$. The usual Kirby moves admit refinements for manifolds equipped with such structures as follows:

- Stabilization: $(L, h) \longrightarrow\left(L \sqcup U_{ \pm 1},(h, 0)\right)$;

- Handle slide: $(L, h) \longrightarrow\left(L^{\prime}, h^{\prime}\right)$ where $L^{\prime}$ is obtained from $L$ by sliding component $L_{i}$ along $L_{j}$ and $h_{k}^{\prime}=h_{k}$ if $k \neq j$ and $h_{j}^{\prime}=h_{j} \mp h_{i}$. Here the sign depends on whether the orientations of $L_{i}$ and $L_{j}$ match or not, respectively;

- Orientation reversal: $(L, h) \longrightarrow\left(L^{\prime}, h^{\prime}\right)$ where $L^{\prime}$ is obtained from $L$ by reversing the orientation of component $L_{i}$ and $h_{j}^{\prime}=h_{j}$ if $j \neq i$ and $h_{i}^{\prime}=-h_{i}$.

For any $h=\left(h_{1}, \ldots, h_{n}\right) \in H^{1}\left(M ; \mathbb{Z}_{d}\right)$ let us define

$$
\tau_{\mathcal{C}}(M, h)=\frac{F_{\mathcal{C}}\left(L\left(\omega_{h_{1}}, \ldots, \omega_{h_{n}}\right)\right)}{F_{\mathcal{C}}\left(U_{1}(\omega)\right)^{b_{+}} F_{\mathcal{C}}\left(U_{-1}(\omega)\right)^{b_{-}}} .
$$

Theorem. For any non-spin d-refinable modular category, $\tau_{\mathcal{C}}(M, h)$ is a topological invariant of the pair $(M, h)$. Moreover,

$$
\tau_{\mathcal{C}}(M)=\sum_{h \in H^{1}\left(M ; \mathbb{Z}_{d}\right)} \tau_{\mathcal{C}}(M, h) .
$$

The proof is based on the following lemma.

Lemma. For any non-spin d-refinable modular category $\mathcal{C}, F_{\mathcal{C}}\left(U_{ \pm 1}\left(w_{u}\right)\right)$ is zero unless $u=0$.

Proof. Just as in the proof of Lemma 6.1, we consider the Hopf link $H_{1,0}\left(\omega_{0}, \omega_{u}\right)$ with linking number -1 and we slide the second component along the first one. We get

$$
\begin{aligned}
F_{\mathcal{C}}\left(U_{1}\left(w_{u}\right)\right) F_{\mathcal{C}}\left(U_{-1}\left(w_{u}\right)\right) & =F_{\mathcal{C}}\left(H_{1,0}\left(\omega_{0}, \omega_{u}\right)\right) \\
& =d F_{\mathcal{C}}\left(H_{1,0}\left(\widetilde{\omega}_{0}, \widetilde{\omega}_{u}\right)\right) \sum_{j=0}^{d-1} e_{d}^{u j}
\end{aligned}
$$

which is zero, unless $u=0$.

6.3. Spin refinements, general case. The proof of Theorem 1 follows from the two previous cases. An $(\widehat{H}, v)$ generalized spin structure $\sigma$ on $M=S^{3}(L)$ is described by a sequence of coefficients $\left(s_{1}, \ldots, s_{n}\right) \in \widehat{H}^{n}$ satisfying a characteristic equation:

$$
\left(s_{1}, \ldots, s_{n}\right)(\widehat{H} \otimes L)=v \otimes\left(L_{11}, \ldots, L_{n n}\right)
$$

Indeed, the Kirby element decomposes using $\widehat{H}$-grading, and the formula for the refined invariant given in (2) still holds. The condition for non vanishing in Lemma 6.1 is $u=v$.

The solution of these equations is a sequence of $\sigma_{i}$ where the index $i$ runs over cyclic components $H_{i} \cong \mathbb{Z}_{d_{i}}$ of $H$. Moreover, either $\sigma_{i} \in \operatorname{Spin}_{d_{i}}(M)$ or $\sigma_{i} \in H^{1}\left(M, \mathbb{Z}_{d_{i}}\right)$, depending on the twist coefficient of the corresponding generator. Combining the two previous theorems we get

$$
\tau_{\mathcal{C}}(M)=\sum_{\sigma} \tau_{\mathcal{C}}(M, \sigma)
$$




\section{Complex SPIN REFinements}

This section is devoted to the proof of Theorem 2. Again, according to the twist coefficients, we will either get an extension of WRT invariants for 3-manifolds equipped with modulo $d$ complex spin structures or with 2-dimensional cohomology classes.

Throughout this section $\mathcal{C}$ is a $2 d$-spin modular category with $d$ even. For any $\sigma \in \mathcal{S}_{d}^{c}(M)$, let us define

$$
\tau_{\mathcal{C}}(M, \sigma)=(-d)^{-n} \sum_{\left(\epsilon_{1}, \ldots, \epsilon_{n}\right) \in \sigma} \frac{F_{\mathcal{C}}\left(L\left(\omega^{\epsilon_{1}}, \ldots, \omega^{\epsilon_{n}}\right)\right)}{F_{\mathcal{C}}\left(U_{1}(\omega)\right)^{b_{+}} F_{\mathcal{C}}\left(U_{-1}(\omega)\right)^{b_{-}}}
$$

where the shorthand $\left(\epsilon_{1}, \ldots, \epsilon_{n}\right) \in \sigma$ means that the summation is taken over all elements of $\left(\mathbb{Z}_{2 d}\right)^{n}$ in the equivalence class of $\sigma$.

Theorem. Let $d$ be an even integer. For any $(2 d)$-spin modular category $\mathcal{C}, \tau_{\mathcal{C}}(M, \sigma)$ is a topological invariant of the pair $(M, \sigma)$.

Proof. In order to prove that $\tau_{\mathcal{C}}(M, \sigma)$ is a topological invariant of the $\operatorname{Spin}_{d}^{c}-$ manifold $(M, \sigma)$, we have to check invariance under the $\operatorname{Spin}_{d}^{c}$ Kirby moves of Theorem 2.4.

We start by checking invariance under the first Kirby move. Let $\left(L^{\prime}, \sigma^{\prime}\right)$ be obtained from $(L, \sigma)$ by a positive stabilization. We have that

$$
\begin{aligned}
\tau_{\mathcal{C}}\left(M, L^{\prime} ; \sigma^{\prime}\right) & =(-d)^{-(n+1)} \sum_{\left(\epsilon_{1}, \ldots, \epsilon_{n+1}\right) \in \sigma^{\prime}} \frac{F_{\mathcal{C}}\left(L\left(\omega^{\epsilon_{1}}, \ldots, \omega^{\epsilon_{n}}\right)\right) F_{\mathcal{C}}\left(U_{1}\left(\omega^{\epsilon_{n+1}}\right)\right)}{F_{\mathcal{C}}\left(U_{1}(\omega)\right)^{b_{+}+1} F_{\mathcal{C}}\left(U_{-1}(\omega)\right)^{b_{-}}} \\
& =(-d)^{-n} \sum_{\left(\epsilon_{1}, \ldots, \epsilon_{n}\right) \in \sigma} \frac{F_{\mathcal{C}}\left(L\left(\omega^{\epsilon_{1}}, \ldots, \omega^{\epsilon_{n}}\right)\right)}{F_{\mathcal{C}}\left(U_{1}(\omega)\right)^{b_{+}} F_{\mathcal{C}}\left(U_{-1}(\omega)\right)^{b_{-}}} \sum_{\epsilon_{n+1} \in 1+2 \mathbb{Z}_{2 d}} \frac{F_{\mathcal{C}}\left(U_{1}\left(\omega^{\epsilon_{n+1}}\right)\right)}{(-d) F_{\mathcal{C}}\left(U_{1}(\omega)\right)} \\
& =\tau_{\mathcal{C}}(M, L ; \sigma) \sum_{x=0}^{2 d-1} \frac{F_{\mathcal{C}}\left(U_{1}\left(\omega^{1+x}\right)\right)}{(-d) F_{\mathcal{C}}\left(U_{1}(\omega)\right)}
\end{aligned}
$$

To compute the sum $\sum_{\substack{x=0 \\ x \text { even }}}^{2 d-1} F_{\mathcal{C}}\left(U_{1}\left(\omega^{1+x}\right)\right)$, we write the dual Kirby color $\omega^{1+x}$ in terms of the refined (graded) Kirby colors $\omega_{i}$ as follows:

$$
\omega^{1+x}=\sum_{i=0}^{2 d-1} \sum_{\lambda \in \Gamma_{i}} e_{2 d}^{(1+x) i}\langle\lambda\rangle \lambda=\sum_{i=0}^{2 d-1} e_{2 d}^{(1+x) i} \omega_{i}
$$

Lemma 6.1 together with the identity $\sum_{\substack{x=0 \\ x \text { even }}}^{2 d-1} e_{2 d}^{(1+x) i}=\left\{\begin{array}{cc}0 & \text { if } i \neq d \\ -d & \text { if } i=d\end{array}\right.$ gives

$$
\begin{aligned}
\sum_{\substack{x=0 \\
x \text { even }}}^{2 d-1} F_{\mathcal{C}}\left(U_{1}\left(\omega^{1+x}\right)\right) & =\sum_{i=0}^{2 d-1} \sum_{\substack{x=0 \\
x \text { even }}}^{2 d-1} e_{2 d}^{(1+x) i} F_{\mathcal{C}}\left(U_{1}\left(\omega_{i}\right)\right) \\
& =-d F_{\mathcal{C}}\left(U_{1}(\omega)\right)
\end{aligned}
$$

so $\tau_{\mathcal{C}}\left(M, L^{\prime} ; \sigma^{\prime}\right)=\tau_{\mathcal{C}}(M, L ; \sigma)$.

Analogously, $\tau_{\mathcal{C}}(M, \sigma)$ is invariant under a negative stabilization. The invariance under the second Kirby move is provided by the dual sliding property. Finally, we must check invariance 
under orientation reversal. For that, let $\left(L^{\prime}, \sigma^{\prime}\right)$ be obtained from $(L, \sigma)$ by changing the orientation of a component $L_{k}$. Without any loss of generality, we may assume that $k=1$ and, just like in the proof of Theorem 4.4, we get $b_{+}^{\prime}=b_{+}, b_{-}^{\prime}=b_{-}$. We have that

$$
\begin{aligned}
\sum_{\left(\epsilon_{1}^{\prime}, \cdots, \epsilon_{n}^{\prime}\right) \in \sigma^{\prime}} F_{\mathcal{C}}\left(L^{\prime}\left(\omega^{\epsilon_{1}^{\prime}}, \cdots, \omega^{\epsilon_{n}^{\prime}}\right)\right) & =\sum_{\left(\epsilon_{1}, \cdots, \epsilon_{n}\right) \in \sigma} F_{\mathcal{C}}\left(L^{\prime}\left(\omega^{-\epsilon_{1}}, \omega^{\epsilon_{2}}, \cdots, \omega^{\epsilon_{n}}\right)\right) \\
& =\sum_{\left(\epsilon_{1}, \cdots, \epsilon_{n}\right) \in \sigma} \sum_{\lambda \in \Gamma} e_{2 d}^{-\epsilon_{1} \operatorname{deg}(\lambda)}\langle\lambda\rangle F_{\mathcal{C}}\left(L^{\prime}\left(\lambda, \omega^{\epsilon_{2}}, \cdots, \omega^{\epsilon_{n}}\right)\right) \\
& =\sum_{\left(\epsilon_{1}, \cdots, \epsilon_{n}\right) \in \sigma} \sum_{\lambda \in \Gamma} e_{2 d}^{\epsilon_{1} \operatorname{deg}\left(\lambda^{*}\right)}\left\langle\lambda^{*}\right\rangle F_{\mathcal{C}}\left(L\left(\lambda^{*}, \omega^{\epsilon_{2}}, \cdots, \omega^{\epsilon_{n}}\right)\right) \\
& =\sum_{\left(\epsilon_{1}, \cdots, \epsilon_{n}\right) \in \sigma} \sum_{\mu \in \Gamma} e_{2 d}^{\epsilon_{1} \operatorname{deg}(\mu)}\langle\mu\rangle F_{\mathcal{C}}\left(L\left(\mu, \omega^{\epsilon_{2}}, \cdots, \omega^{\epsilon_{n}}\right)\right) \\
& =\sum_{\left(\epsilon_{1}, \cdots, \epsilon_{n}\right) \in \sigma} F_{\mathcal{C}}\left(L\left(\omega^{\epsilon_{1}}, \cdots, \omega^{\epsilon_{n}}\right)\right) .
\end{aligned}
$$

The first equality above is a consequence of the fact that $\left(L_{i j}^{\prime}\right)={ }^{t} S\left(L_{i j}\right) S$, for $S=\operatorname{diag}(-1,1, \cdots, 1)$ while the third equality is an immediate application of Corollary 4.1.1. This concludes the proof.

7.1. Homological refinements. Let $d$ be a positive integer and $\mathcal{C}$ be a non-spin $d$-refinable modular category.

The group $H_{1}\left(M ; \mathbb{Z}_{d}\right)$ is described combinatorially as the set $\left(\mathbb{Z}_{d}\right)^{n} / \operatorname{Im} L$. The Kirby moves for the pair $(M, h)$ where $M$ is obtained by surgery on a link $L$ and $h \in H_{1}\left(M ; \mathbb{Z}_{d}\right)$ can be described as follows:

- Stabilization: $(L, h) \longrightarrow\left(L \sqcup U_{ \pm 1},(h, 0)\right)$;

- Handle slide: $(L, h) \longrightarrow\left(L^{\prime}, h^{\prime}\right)$ where $L^{\prime}$ is obtained from $L$ by sliding component $L_{i}$ along $L_{j}$ and $h_{k}^{\prime}=h_{k}$ if $k \neq i$ and $h_{i}^{\prime}=h_{i} \pm h_{j}$. Here the sign depends on whether the orientations of $L_{i}$ and $L_{j}$ match or not, respectively;

- Orientation reversal: $(L, h) \longrightarrow\left(L^{\prime}, h^{\prime}\right)$ where $L^{\prime}$ is obtained from $L$ by changing the orientation of component $L_{i}$ and $h_{j}^{\prime}=h_{j}$ if $j \neq i$ and $h_{i}^{\prime}=-h_{i}$.

For any $h \in H_{1}\left(M ; \mathbb{Z}_{d}\right)$ let us define

$$
\tau_{\mathcal{C}}(M, h)=d^{-n} \sum_{\left(\epsilon_{1}, \ldots, \epsilon_{n}\right) \in h} \frac{F_{\mathcal{C}}\left(L\left(\omega^{\epsilon_{1}}, \ldots, \omega^{\epsilon_{n}}\right)\right)}{F_{\mathcal{C}}\left(U_{1}(\omega)\right)^{b^{+}} F_{\mathcal{C}}\left(U_{-1}(\omega)\right)^{b_{-}}}
$$

where the shorthand $\left(\epsilon_{1}, \ldots, \epsilon_{n}\right) \in h$ means that the summation is taken over all elements of $\left(\mathbb{Z}_{d}\right)^{n}$ in the equivalence class of $h$.

Theorem. For any non-spin d-refinable modular category $\mathcal{C}, \tau_{\mathcal{C}}(M, h)$ is a topological invariant of the pair $(M, h)$.

Proof. In order to prove that $\tau_{\mathcal{C}}(M, h)$ is a topological invariant of the manifold $(M, h)$, we have to check invariance under the Kirby moves listed above. 
We start by checking invariance under the first Kirby move. Let $\left(L^{\prime}, h^{\prime}\right)$ be obtained from $(L, h)$ by a positive stabilization.

$$
\begin{aligned}
\tau_{\mathcal{C}}\left(M, L^{\prime} ; h^{\prime}\right) & =d^{-(n+1)} \sum_{\left(\epsilon_{1}, \ldots, \epsilon_{n+1}\right) \in h^{\prime}} \frac{F_{\mathcal{C}}\left(L\left(\omega^{\epsilon_{1}}, \ldots, \omega^{\epsilon_{n}}\right)\right) F_{\mathcal{C}}\left(U_{1}\left(\omega^{\epsilon_{n+1}}\right)\right)}{F_{\mathcal{C}}\left(U_{1}(\omega)\right)^{b_{+}+1} F_{\mathcal{C}}\left(U_{-1}(\omega)\right)^{b_{-}}} \\
& =d^{-n} \sum_{\left(\epsilon_{1}, \ldots, \epsilon_{n}\right) \in h} \frac{F_{\mathcal{C}}\left(L\left(\omega^{\epsilon_{1}}, \ldots, \omega^{\epsilon_{n}}\right)\right)}{F_{\mathcal{C}}\left(U_{1}(\omega)\right)^{b}+F_{\mathcal{C}}\left(U_{-1}(\omega)\right)^{b_{-}}} \sum_{i=0}^{d-1} \frac{F_{\mathcal{C}}\left(U_{1}\left(\omega^{i}\right)\right)}{d F_{\mathcal{C}}\left(U_{1}(\omega)\right)} \\
& =\tau_{\mathcal{C}}(M, L ; h) \frac{F_{\mathcal{C}}\left(U_{1}\left(\sum_{i=0}^{d-1} \omega^{i}\right)\right)}{d F_{\mathcal{C}}\left(U_{1}(\omega)\right)} .
\end{aligned}
$$

We compute

$$
\begin{aligned}
\sum_{i=0}^{d-1} \omega^{i} & =\sum_{i=0}^{d-1} \sum_{j=0}^{d-1} \sum_{\lambda \in \Gamma_{j}} e_{d}^{i j}\langle\lambda\rangle \lambda \\
& =\sum_{j=0}^{d-1} \sum_{\lambda \in \Gamma_{j}}\left(\sum_{i=0}^{d-1} e_{d}^{i j}\right)\langle\lambda\rangle \lambda \\
& =d \cdot \omega_{0}
\end{aligned}
$$

since $\sum_{i=0}^{d-1} e_{d}^{i j}=0$ unless $j=0$. Using Lemma 6.2 we get that $F_{\mathcal{C}}\left(U_{1}(\omega)\right)=F_{\mathcal{C}}\left(U_{1}\left(\omega_{0}\right)\right)$ and therefore $\tau_{\mathcal{C}}\left(M, L^{\prime} ; h^{\prime}\right)=\tau_{\mathcal{C}}(M, L ; h)$.

Analogously, $\tau_{\mathcal{C}}(M, h)$ is invariant under a negative stabilization. The invariance under the second Kirby move is provided by the dual sliding property. Finally, we must check invariance under orientation reversal. For that, let $\left(L^{\prime}, h^{\prime}\right)$ be obtained from $(L, h)$ by changing the orientation of a component $L_{k}$. Without any loss of generality, we may assume that $k=1$ and, just like in the proof of Theorem 4.4, we get $b_{+}^{\prime}=b_{+}, b_{-}^{\prime}=b_{-}$. Since $h^{\prime}=\left(-h_{1}, h_{2}, \cdots, h_{n}\right)$, it follows that

$$
\begin{aligned}
\sum_{\left(\epsilon_{1}^{\prime}, \cdots, \epsilon_{n}^{\prime}\right) \in h^{\prime}} F_{\mathcal{C}}\left(L^{\prime}\left(\omega^{\epsilon_{1}^{\prime}}, \cdots, \omega^{\epsilon_{n}^{\prime}}\right)\right) & =\sum_{\left(\epsilon_{1}, \cdots, \epsilon_{n}\right) \in h} F_{\mathcal{C}}\left(L^{\prime}\left(\omega^{-\epsilon_{1}}, \omega^{\epsilon_{2}}, \cdots, \omega^{\epsilon_{n}}\right)\right) \\
& =\sum_{\left(\epsilon_{1}, \cdots, \epsilon_{n}\right) \in h} \sum_{\lambda \in \Gamma} e_{d}^{-\epsilon_{1} \operatorname{deg}(\lambda)}\langle\lambda\rangle F_{\mathcal{C}}\left(L^{\prime}\left(\lambda, \omega^{\epsilon_{2}}, \cdots, \omega^{\epsilon_{n}}\right)\right) \\
& =\sum_{\left(\epsilon_{1}, \cdots, \epsilon_{n}\right) \in h} \sum_{\lambda \in \Gamma} e_{d}^{\epsilon_{1} \operatorname{deg}\left(\lambda^{*}\right)}\left\langle\lambda^{*}\right\rangle F_{\mathcal{C}}\left(L\left(\lambda^{*}, \omega^{\epsilon_{2}}, \cdots, \omega^{\epsilon_{n}}\right)\right) \\
& =\sum_{\left(\epsilon_{1}, \cdots, \epsilon_{n}\right) \in h} \sum_{\mu \in \Gamma} e_{d}^{\epsilon_{1} \operatorname{deg}(\mu)}\langle\mu\rangle F_{\mathcal{C}}\left(L\left(\mu, \omega^{\epsilon_{2}}, \cdots, \omega^{\epsilon_{n}}\right)\right) \\
& =\sum_{\left(\epsilon_{1}, \cdots, \epsilon_{n}\right) \in h} F_{\mathcal{C}}\left(L\left(\omega^{\epsilon_{1}}, \cdots, \omega^{\epsilon_{n}}\right)\right) .
\end{aligned}
$$

The first equality above is a consequence of the fact that $\left(L_{i j}^{\prime}\right)={ }^{t} S\left(L_{i j}\right) S$, for $S=\operatorname{diag}(-1,1, \cdots, 1)$ while the third equality is an immediate application of Corollary 4.1.1. This concludes the proof. 


\section{Decomposition formula}

This section is devoted to the proof of Theorem 3. Reader interested in the case $\operatorname{gcd}(m, \delta)=1$ only can skip this section and consult an easy direct argument in Appendix.

Throughout this section $d$ is any positive integer, it needs not to be even.

8.1. Strategy of the proof. Let us recall the setting. We assume that $\mathcal{C}$ be a modular category with cyclic group of invertible objects $G=\langle t\rangle$. Let $\operatorname{deg}(t)=\delta \in \mathbb{Z}_{d} \cong \widehat{G}$ and $m \delta=d$. Moreover, let us split $\delta=\alpha \beta$, $\operatorname{such}$ that $\operatorname{gcd}(\beta, \alpha m)=1, \alpha \equiv m(\bmod 2)$.

For any $V \in \operatorname{Ob}(\mathcal{C})$ we can choose $\chi_{V}(t)=\kappa^{\operatorname{deg}(V)}$ where $\kappa$ is a primitive $d$-th root of unity. Let us fix the generator $t$, so that $\kappa=e^{\frac{i 2 \pi}{d}}$. The twist coefficient for $t$ is $\theta_{t}=b_{t, t}\langle t\rangle$ and satisfies $\theta_{t}^{2}=\kappa^{\delta}=e^{\frac{i 2 \pi}{m}}$. We consider the subgroup of invertible objects $H=\left\langle t^{m}\right\rangle \cong \mathbb{Z}_{\delta}$. Clearly, $H \subset \mathcal{C}_{0}$, so the modular category $\mathcal{C}$ is $\delta$-refinable. The twist coefficient for the generator $t^{m}$ is $\theta_{t^{m}}=b_{t, t}^{m^{2}}\langle t\rangle^{m}$. It is equal to -1 if $\delta$ is even, $m$ is odd and either $\langle t\rangle=1$ and $b_{t, t}=\theta_{t}=e^{\frac{i \pi}{m}}$, or $\langle t\rangle=-1$ and $b_{t, t}=-\theta_{t}=-e^{\frac{i \pi}{m}}$; it is equal to 1 in all other cases. The modular category $\mathcal{C}$ is $\delta$-spin if $\delta$ is even, $m$ is odd and $\theta_{t}$ has order $2 m$, and $\delta$-cohomological in all other cases.

We now present the idea of the proof of the decomposition statement. We define a tensor category $\mathcal{C}^{\prime}$ with simple objects represented by $\Gamma^{\prime}=\Gamma \times \mathbb{Z}_{\alpha}$. The tensor product in $\mathcal{C}^{\prime}$ mimics central extension of groups using 2-cocycles. We lift the map deg $: \Gamma \rightarrow \mathbb{Z}_{d}$ into a map $f: \Gamma \rightarrow$ $\mathbb{Z}_{d \alpha}$, which plays the role of a section. Further, we extend $f$ into a map $\operatorname{Ob}\left(\mathcal{C}^{\prime}\right) \rightarrow \mathbb{Z}_{\alpha d}$, such that

$$
f(V, k)=f(V)+d k \quad \text { for } \quad(V, k) \in \Gamma^{\prime} .
$$

Given two elements $(V, k)$ and $(W, l)$ of $\Gamma^{\prime}$ we define their tensor product as $(V \otimes W, k+l)$. We allow in $\mathcal{C}^{\prime}$ direct sums of objects with homogenous $f$ value.

For $X=(V, k) \in \operatorname{Ob}\left(\mathcal{C}^{\prime}\right)$ and $Y=(W, l) \in \operatorname{Ob}\left(\mathcal{C}^{\prime}\right)$ we set

$$
\operatorname{Hom}_{\mathcal{C}^{\prime}}(X, Y)=\left\{\begin{array}{l}
0 \text { if } f(X) \neq f(Y) \\
\operatorname{Hom}_{\mathcal{C}}(V, W) \text { else. }
\end{array}\right.
$$

The category $\mathcal{C}^{\prime}$ is a tensor category over $\mathbf{k}$ with unit object $(\mathbf{1}, 0)$ and compatible duality. Note that $\left(V^{*}, l\right)$ is a left and right dual for $(V, k)$ if $l$ is choosen so that $f\left(V^{*}, l\right)=-f(V, k)$.

Proposition. The category $\mathcal{C}^{\prime}$ is semisimple with $\Gamma^{\prime}$ as representative set of simple objects. The group of invertible objects is $G^{\prime} \cong \mathbb{Z}_{d} \times \mathbb{Z}_{\alpha}$, generated by $(t, 0)$ and $(\mathbf{1}, 1)$.

Proof. The object $(V, j)$ is invertible in $\mathcal{C}^{\prime}$ if and only if $V$ is invertible in $\mathcal{C}$. We deduce the last statement. To prove semisimplicity, it is enough to decompose the tensor product of two objects objects $(V, j),\left(V^{\prime}, j^{\prime}\right)$ in $\Gamma^{\prime}$. We have in the category $\mathcal{C}$ a decomposition

$$
\operatorname{Id}_{V} \otimes \operatorname{Id}_{V^{\prime}}=\sum_{W} \sum_{\nu \in I_{V, V^{\prime}}^{W}} b_{\nu} \operatorname{Id}_{W} a_{\nu}
$$

We set $\chi_{V, V^{\prime}}^{W}=\left(f(V)+f\left(V^{\prime}\right)-f(W)\right) / d$. Then we have in the category $\mathcal{C}^{\prime}$ the following decomposition

$$
\operatorname{Id}_{(V, j)} \otimes \operatorname{Id}_{\left(V^{\prime}, j^{\prime}\right)}=\sum_{W} \sum_{\nu \in I_{V, V^{\prime}}^{W}} b_{\nu} \operatorname{Id}_{\left(W, j+j^{\prime}+\chi_{V, V^{\prime}}^{W}\right)} a_{\nu}
$$


Further, let us give $\mathcal{C}^{\prime}$ a ribbon structure which twists the one given on $\mathcal{C}$. The braiding is given by a formula

$$
b_{(V, k),(W, l)}^{\prime}=\xi^{-f(V, k) f(W, l)} b_{V, W},
$$

with appropriate choice of a root of unity $\xi$ whose order $2 \alpha d$ if $d$ is even and $\alpha d$ if $d$ is odd. Using duality, the twist is then given by

$$
\theta_{(V, k)}^{\prime}=\xi^{-f(V, k)^{2}} \theta_{V}
$$

The corresponding colored link invariants $F=F_{\mathcal{C}}$ and $F^{\prime}=F_{\mathcal{C}^{\prime}}$ are equal up to a power of $\xi$ which is computed from map $f$ and linking numbers. Note that $\xi$ is choosen such that $\alpha m$ elements of $G^{\prime}$ become transparent.

The $G^{\prime}$-category $\mathcal{C}^{\prime}$ is premodular and can be modularized as described in [6, 1]. Simple objects in the modularization $\widetilde{\mathcal{C}}$ are obtained from those of $\mathcal{C}^{\prime}$ quotienting by a free action. The set $\tilde{\Gamma}$ of simple objects in $\tilde{\mathcal{C}}$ has cardinality $|\Gamma| / m$. Below we give a detailed proof of the decomposition formula in the spin case, the cohomological cases can be proven similarly.

Proof of Theorem 3, spin case. We consider here the spin case, which means that $d$ is even, $m$ is odd and the twist $\theta_{t}$ has order $2 m$. The generator $t$ can be choosen so that $\theta_{t}=e^{\frac{i \pi}{m}}$. Let $\xi=e^{\frac{i \pi l}{\alpha^{2} m}}$ with $\beta^{2} l \equiv 1+\alpha^{2} m \bmod 2 \alpha^{2} m$. Note that $\xi^{\alpha d=1}$ so that the modified braiding is well defined.

The braiding coefficients for the generators of $G^{\prime}$ are:

$$
\begin{gathered}
b_{X,(t, 0)}^{\prime}=\left(\kappa \xi^{-2 \delta}\right)^{f(X)}=e^{\frac{2 i \pi\left(1-l \beta^{2}\right) f(X)}{d}}, \\
b_{X,(\mathbf{1}, 1)}^{\prime}=\left(\xi^{-2 d}\right)^{f(X)}=e^{\frac{2 i \pi l f(X) \beta}{\alpha}} .
\end{gathered}
$$

The twist coefficients are

$$
\begin{gathered}
\theta_{(t, 0)}^{\prime}=e^{\frac{i \pi}{m}} e^{-\frac{i \pi l \beta^{2}}{m}}=-1, \\
\theta_{(\mathbf{1}, 1)}^{\prime}=e^{-\frac{i \pi l d^{2}}{\alpha^{2} m}}=1 .
\end{gathered}
$$

It follows that the group of transparent objects is generated by $(t, 0)^{\beta}$ which has trivial twist and quantum dimension 1 . Applying the results $[6,1]$ we see that the category $\mathcal{C}^{\prime}$ is modularizable, i.e. that there exists a modular category $\widetilde{\mathcal{C}}$ and a dominant ribbon functor $\mathcal{C}^{\prime} \rightarrow \widetilde{\mathcal{C}}$. Here the group of transparent objects acts freely on the set $\Gamma^{\prime}$ of simple objects in $\mathcal{C}^{\prime}$. This is proved using the map $f$ and the fact that $f\left((t, 0)^{\beta}\right)=\beta \delta$ has order $\alpha m$ which is the order of $(t, 0)^{\beta}$. Hence, the simple objects $\widetilde{\Gamma}$ in $\widetilde{\mathcal{C}}$ are represented by cosets in $\Gamma^{\prime}$ under this free action.

In the category $\widetilde{\mathcal{C}}$ the group of invertible objects is $\widetilde{G}=\langle(t, 0),(\mathbf{1}, 1)\rangle \cong \mathbb{Z}_{\beta} \times \mathbb{Z}_{\alpha} \cong \mathbb{Z}_{\delta}$. The twist coefficient for $(t, 0)$ is equal to -1 , so the category $\widetilde{\mathcal{C}}$ is $\delta$-spin. Denote by $\omega^{\prime}$ the Kirby element in $\mathcal{C}^{\prime}$ which represents $\alpha m$ times the Kirby element $\widetilde{\omega}$ in $\widetilde{\mathcal{C}}$. We write the graded decomposition

$$
\omega^{\prime}=\sum_{c \in \mathbb{Z}_{\delta}} \omega_{c}^{\prime}=\alpha \sum_{c \in \mathbb{Z}_{\delta}} \omega_{c}
$$

so that

$$
\omega_{c}^{\prime}=\sum_{\substack{\gamma \in \mathbb{Z}_{\alpha d} d \\ \gamma \equiv c \bmod \delta}} \sum_{\substack{X \in \Gamma^{\prime} \\ f(X)=\gamma}}\langle X\rangle X=\sum_{\gamma \equiv c \bmod \delta} \omega_{\mid f=\gamma}^{\prime} .
$$


Moreover, for any $\mathbb{Z}_{\alpha d} \ni \gamma \equiv c \bmod \delta$, the Kirby color $\widetilde{\omega}_{c}$ in $\widetilde{\mathcal{C}}$ is represented by $\omega_{\mid f=\gamma}^{\prime}$, i.e.

$$
\widetilde{\omega}_{c}=\omega_{\mid f=\gamma}^{\prime}=\frac{1}{\alpha m} \omega_{c}^{\prime} .
$$

Indeed, the set $\omega_{\mid f=\gamma}^{\prime}$ consists of all $(V, k) \in \Gamma^{\prime}$ such that $f(V)=\gamma-k d \bmod \alpha d$. There are $|\Gamma| / d$ such elements. Acting with $\left(t^{\beta}, 0\right)$ we can shift the degree of solutions by $\delta$. In this way we obtain all $(V, k) \in \Gamma^{\prime}$ with $\operatorname{deg}(V)=c \bmod \delta$. Taking the quotient by this action we get $\tilde{\omega}_{c}$.

It makes sense to evaluate both Reshetikhin-Turaev ribbon functors $F=F_{\mathcal{C}}$, and $F^{\prime}=F_{\mathcal{C}^{\prime}}$ on $\mathcal{C}^{\prime}$ colored links. Let $M=S^{3}(L)$ be a 3 -manifold given by surgery on the $n$-component link $L$ with signature $\left(b_{+}, b_{-}\right)$, and $\sigma \in \operatorname{Spin}_{\delta}(M)$ represented by coefficients $c_{j} \in \mathbb{Z}_{\delta}, 1 \leq j \leq n$. For objects $X_{j} \in \Gamma^{\prime}, 1 \leq j \leq n$, we have

$$
F\left(L\left(X_{1}, \ldots, X_{n}\right)\right)=\xi^{t} f(X) L f(X) F^{\prime}\left(L\left(X_{1}, \ldots, X_{n}\right)\right)
$$

where ${ }^{t} f(X) L f(X)=\sum_{i, j} L_{i j} f\left(X_{i}\right) f\left(X_{j}\right)$. Note that the left hand side is invariant under action of $(t, 0)^{\beta}$ on objects and can be used for the evaluation of the reduced invariant $\tau_{\widetilde{\mathcal{C}}}(M, \sigma)$ which we want to compare with $\tau_{\mathcal{C}}(M, \sigma)$. We have

$$
\begin{aligned}
& \tau_{\mathcal{C}}(M, \sigma)=\frac{F\left(L\left(\omega_{c_{1}}, \ldots, \omega_{c_{n}}\right)\right)}{\left(F\left(U_{1}\left(\omega_{\delta / 2}\right)\right)\right)^{b_{+}}\left(F\left(U_{-1}\left(\omega_{\delta / 2}\right)\right)\right)^{b_{-}}} \\
& =\frac{\alpha^{-n+b_{+}+b_{-}} F\left(L\left(\omega_{c_{1}}^{\prime}, \ldots, \omega_{c_{n}}^{\prime}\right)\right)}{\left(F\left(U_{1}\left(\omega_{\delta / 2}^{\prime}\right)\right)\right)^{b_{+}}\left(F\left(U_{-1}\left(\omega_{\delta / 2}^{\prime}\right)\right)\right)^{b_{-}}} \\
& F\left(L\left(\omega_{c_{1}}^{\prime}, \ldots, \omega_{c_{n}}^{\prime}\right)\right)=\sum_{\substack{\gamma \in\left(\mathbb{Z}_{\alpha d}\right)^{n} \\
\gamma_{i}=c_{i} \bmod \delta}} F\left(L\left(\omega_{\mid f=\gamma_{1}}^{\prime}, \ldots, \omega_{\mid f=\gamma_{n}}^{\prime}\right)\right) \\
& =\sum_{\substack{\gamma \in\left(\mathbb{Z}_{\alpha d}\right)^{n} \\
\gamma_{i} \equiv c_{i} \bmod \delta}} \xi^{t} \gamma L \gamma F^{\prime}\left(L\left(\omega_{\mid f=\gamma_{1}}^{\prime}, \ldots, \omega_{\mid f=\gamma_{n}}^{\prime}\right)\right) \\
& =\sum_{\substack{\gamma \in\left(\mathbb{Z}_{\alpha d)}\right) \\
\gamma_{i} \equiv c_{i} \bmod \delta}} \xi^{t} \gamma L \gamma F^{\prime}\left(L\left(\tilde{\omega}_{c_{1}}, \ldots, \tilde{\omega}_{c_{n}}\right)\right) \\
& =F^{\prime}\left(L\left(\tilde{\omega}_{c_{1}}, \ldots, \tilde{\omega}_{c_{n}}\right)\right) \sum_{\substack{\gamma \in\left(\mathbb{Z}_{\alpha d}\right)^{n} \delta \\
\gamma_{i} \equiv c_{i} \bmod \delta}} \xi^{t} \gamma L \gamma
\end{aligned}
$$

After normalization we get

$$
\begin{aligned}
\tau_{\mathcal{C}}(M, \sigma) & =\alpha^{-b_{1}(M)} \tau_{\widetilde{\mathcal{C}}}(M, \sigma) g^{-b_{+}}(\bar{g})^{-b_{-}} \sum_{\substack{\left.\gamma \in \mathbb{Z}_{\alpha d}\right)^{n} \\
\gamma \equiv c \bmod \delta}} \xi^{t} \gamma L \gamma \text { where } \\
g & =\sum_{\substack{\gamma \in \mathbb{Z}_{\alpha d} \\
\gamma \equiv \delta / 2 \bmod \delta}} \xi^{\gamma^{2}}
\end{aligned}
$$

and $\bar{g}$ is the complex conjugate. One can check, following the graded construction in Section 6 that the formula

$$
\tau_{\xi}^{\mathrm{MOO}}(M, \sigma)=g^{-b_{+}}(\bar{g})^{-b_{-}} \sum_{\gamma \equiv c \bmod \delta} \xi^{t} \gamma L \gamma
$$

defines an invariant of $(M, \sigma)$. We conclude

$$
\tau_{\mathcal{C}}(M, \sigma)=\alpha^{-b_{1}(M)} \tau_{\widetilde{\mathcal{C}}}(M, \sigma) \tau_{\xi}^{\mathrm{MOO}}(M, \sigma) .
$$


In the case $\operatorname{gcd}(m, \delta)=1$, we have $\alpha=1$ and $\sum_{j} L_{i j} \gamma_{j}=\frac{\delta}{2} L_{i i} \bmod \delta$. Furthermore, we can assume that our surgery presentation has even linking matrix (the obstruction given by the spin cobordism group vanishes), so that $c \in \operatorname{Ker} L \bmod \delta$. Decomposing $\gamma=\gamma_{0}+\delta x$ with $\gamma_{0} \in \operatorname{Ker} L$ and $x \in \mathbb{Z}_{m}$ we see that

$$
\sum_{\gamma \equiv c \bmod \delta} \xi^{t} \gamma L \gamma=\sum_{x \in\left(\mathbb{Z}_{m}\right)^{n}} \xi^{t\left(\gamma_{0}+\delta x\right) L\left(\gamma_{0}+\delta x\right)}=\sum_{x \in\left(\mathbb{Z}_{m}\right)^{n}} \xi^{t(\delta x) L(\delta x)}=\sum_{x \in\left(\mathbb{Z}_{m}\right)^{n}} \xi^{t} x L x
$$

does not depend on $c$. Summing over $\sigma$ we get

$$
\tau_{\mathcal{C}}(M)=\tau_{\widetilde{\mathcal{C}}}(M) \tau_{\xi}^{\mathrm{MOO}}(M) .
$$

\section{APPENDIX}

Here we give a simple direct proof of Theorem 3 in the case when $\operatorname{gcd}(m, \delta)=1$. For readers convenience, we repeat the statement.

Theorem. Let $\mathcal{C}$ be a modular $d$-category with the group $G=\langle t\rangle$ of invertible objects, such that $\operatorname{deg} t=\delta, \operatorname{gcd}(\delta, d / \delta)=1$. Then there are exists a subcategory $\widetilde{\mathcal{C}} \subset \mathcal{C}$ and a root of unity $\xi$, such that for any closed orientable 3-manifold $M$

$$
\tau_{\mathcal{C}}(M)=\tau_{\widetilde{\mathcal{C}}}(M) \tau_{\xi}^{\mathrm{MMO}}(M) .
$$

Let $m$ be such that $\delta m=d$ and we set

$$
\widetilde{\Gamma}:=\{\lambda \in \Gamma \mid \operatorname{deg}(\lambda)=0 \quad(\bmod m)\} .
$$

Let $\widetilde{\mathcal{C}}$ be the full ribbon subcategory of $\mathcal{C}$ generated by $\widetilde{\Gamma}$ and $\widetilde{\omega}$ be the corresponding Kirby color. Let $K=\left\langle t^{m}\right\rangle$. In this situation $K$ has order $\delta$ and $\widetilde{\Gamma}=\Gamma_{0} \amalg \Gamma_{m} \amalg \cdots \amalg \Gamma_{(\delta-1) m}$. Moreover, consider $\eta \in \mathbb{C}$ such that

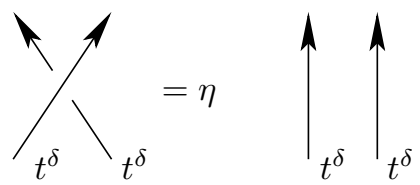

We will need the following proposition.

Proposition. Let $\xi=\eta\langle t\rangle^{\delta}$. Then we have the following equalities of morphisms:

(a) for any $\lambda \in \widetilde{\Gamma}$ and $k \in\{0, \cdots, m-1\}$

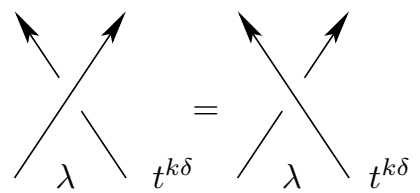

(b) for any $k, s \in\{0, \cdots, m-1\}$

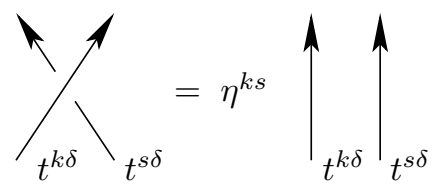


(c) for any $k, s \in\{0, \cdots, m-1\}$

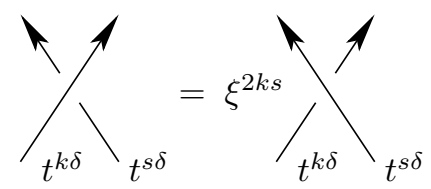

(d) for any $k \in\{0, \cdots, m-1\}$, the twist coefficient $\theta_{t^{k \delta}}=\xi^{k^{2}}$.

The proof is a straightforward computation using that $\langle t\rangle= \pm 1$ and $\xi^{2}=\eta^{2}=e_{d}^{\delta^{3}}$.

Lemma. The category $\widetilde{\mathcal{C}}$ is modular and reduced, in the sense that it is $\widetilde{G}-$ refinable.

Proof. Clearly, $1 \in \widetilde{\Gamma}$ since $\operatorname{deg}(1)=0$. Duality axiom holds since for $\lambda \in \widetilde{\Gamma}, \operatorname{deg}\left(\lambda^{*}\right)=-\operatorname{deg}(\lambda)$ and hence $\lambda^{*} \in \widetilde{\Gamma}$. Domination follows trivially from the same property of $\mathcal{C}$. It remains to prove the non-degeneracy.

Observe that the Kirby color $\omega$ decomposes as the sum

$$
\omega=\sum_{i=0}^{m-1}\langle t\rangle^{i \delta} t^{i \delta} \widetilde{\omega}
$$

since $(\delta, m)=1$. Decomposing $\omega$ as above and using Proposition 8.1 (a) we get for any $\lambda \in \widetilde{\Gamma}$ the following equalities

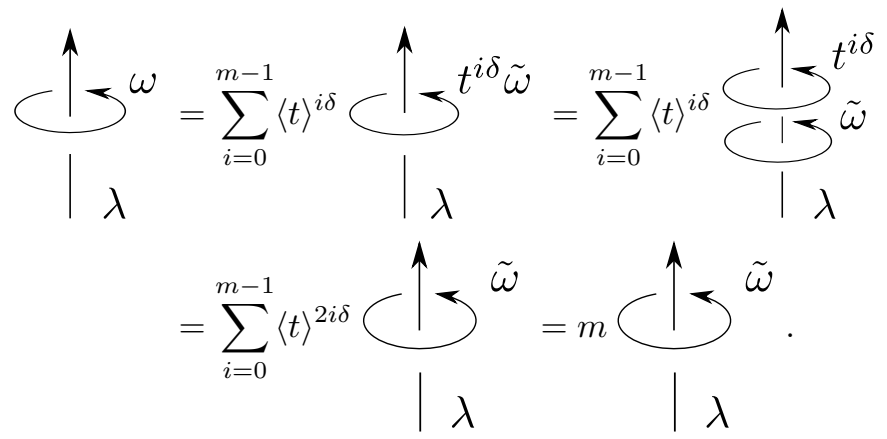

The killing property combined with Proposition 4.3 implies non-degeneracy.

Proof of Theorem. Let $M$ be presented by an oriented framed link $L=\left(L_{1}, \cdots, L_{n}\right)$ with all components $L_{i}$ unknotted (such a link always exists, see [12]). Then, the invariant

$$
\tau_{\mathcal{C}}(M)=\frac{F_{\mathcal{C}}(L(\omega, \cdots, \omega))}{\left(F_{\mathcal{C}}\left(U_{1}(\omega)\right)\right)^{b_{+}}\left(F_{\mathcal{C}}\left(U_{-1}(\omega)\right)\right)^{b_{-}}} .
$$

If we replace $\omega=\sum_{i=0}^{m-1}\langle t\rangle^{i \delta} t^{i \delta} \widetilde{\omega}$ in $F_{\mathcal{C}}(L(\omega, \cdots, \omega))$ and apply Proposition (a) we obtain:

$$
F_{\mathcal{C}}(L(\omega, \cdots, \omega))=F_{\widetilde{\mathcal{C}}}(L(\widetilde{\omega}, \cdots, \widetilde{\omega})) \sum_{i_{1}, \cdots, i_{n}=0}^{m-1}\langle t\rangle^{\left(i_{1}+\cdots+i_{n}\right) \delta} F_{\mathcal{C}}\left(L\left(t^{i_{1} \delta}, \cdots, t^{i_{n} \delta}\right)\right) .
$$

In particular, for $\epsilon= \pm 1$, we have

$$
F_{\mathcal{C}}\left(U_{\epsilon}(\omega)\right)=F_{\widetilde{\mathcal{C}}}\left(U_{\epsilon}(\widetilde{\omega})\right) \sum_{i=0}^{m-1}\langle t\rangle^{i \delta} F_{\mathcal{C}}\left(U_{\epsilon}\left(t^{i \delta}\right)\right) .
$$

Given the link $L$ with components colored by $t^{i_{1} \delta}, \cdots, t^{i_{n} \delta}, F_{\mathcal{C}}\left(L\left(t^{i_{1} \delta}, \cdots, t^{i_{n} \delta}\right)\right)$ can be computed as follows: first we make each component of $L$ zero framed, then we unlink the components 
(using Proposition (c) and (d) as many times as necessary). Finally, we obtain $n$ disjoint and unlinked copies of the zero framed unknot with colors $t^{i_{1} \delta}, \cdots, t^{i_{n} \delta}$ and the relation:

$$
F_{\mathcal{C}}\left(L\left(t^{i_{1} \delta}, \cdots, t^{i_{n} \delta}\right)\right)=\langle t\rangle^{\left(i_{1}+\cdots+i_{n}\right) \delta} \cdot \xi^{\left(i_{1}, \cdots, i_{n}\right) L\left(i_{1}, \cdots, i_{n}\right)^{t}},
$$

where $\left(L_{i j}\right)$ is the linking matrix of $L$. Similarly

$$
F_{\mathcal{C}}\left(U_{\epsilon}\left(t^{i \delta}\right)\right)=\langle t\rangle^{i \delta} \cdot \xi^{\epsilon i^{2}}
$$

and the Reshetikin-Turaev invariant decomposes as

$$
\tau_{\mathcal{C}}(M)=\tau_{\widetilde{\mathcal{C}}}(M) \frac{\sum_{l \in\left(\mathbb{Z}_{m}\right)^{n}} \xi^{t} l L l}{\left(\sum_{i \in \mathbb{Z}_{m}} \xi^{i^{2}}\right)^{b_{+}}\left(\sum_{i \in \mathbb{Z}_{m}} \xi^{-i^{2}}\right)^{b_{-}}} .
$$

Note that for $d$ even, $\xi$ is an $m$ th root of unity if $m$ is odd and a $2 m$ th root of unity if $m$ is even and according to [16],

$$
\tau_{\xi}^{\mathrm{MMO}}(M)=\frac{\sum_{l \in\left(\mathbb{Z}_{m}\right)^{n}} \xi^{t} l L l}{\left(\sum_{i \in \mathbb{Z}_{m}} \xi^{i^{2}}\right)^{b_{+}}\left(\sum_{i \in \mathbb{Z}_{m}} \xi^{-i^{2}}\right)^{b_{-}}}
$$

is a topological invariant of $M$ independent on the choice of $L$, known as the Murakami-OhtsukiOkada invariant.

\section{REFERENCES}

[1] A. Beliakova, and C. Blanchet. Modular categories of type B,C and D, Comment. Math. Helv. 76 (2001), $467-500$.

[2] C. Blanchet. Invariants of three-manifolds with spin structure, Comment. Math. Helv. 67 (1992), $406-427$.

[3] C. Blanchet. Hecke algebras, modular categories and 3- manifolds quantum invariants, Topology 39 (2000), 193-223.

[4] C. Blanchet. A spin decomposition of the Verlinde formulas for type A modular categories, Comm. in Math. Physics 257, (2005), 1 -28.

[5] C. Blanchet, N. Habegger, G. Masbaum and P. Vogel. Three-manifold invariants derived from the Kauffman bracket, Topology 31 No 4, (1992) 685-699.

[6] A. Bruguières. Premodular categories, modularisations and invariants of 3-manifolds. (Catégories prémodulaires, modularisations et invariants des variétés de dimension 3.) Math. Ann. 316, No.2, (2000) 215-236.

[7] F. Deloup, and G. Massuyeau. Quadratic functions and complex spin structures on three-manifolds, Topology 44(3) (2005), 509-555.

[8] R. Kirby. A calculus for framed links in $S^{3}$, Invent. Math. 45(1) (1978), 36-58.

[9] R. Kirby, and P. Melvin. The 3-manifold invariants of Witten and Reshetikhin-Turaev for $\mathfrak{s l}(2, \mathbb{C})$, Invent. Math. 105 (1991), 473-545.

[10] T. Le. Quantum invariants of 3-manifolds: integrality, splitting, and perturbative expansion

[11] W.B.R. Lickorish. Three-manifold invariants and the Temperley-Lieb algebra, Ann. of Math. 290 (1991), $657-670$.

[12] W.B.R. Lickorish. An Introduction to Knot Theory, Graduate Texts in Mathematics, Springer 175 (1997).

[13] S. Mac Lane. Categories for the Working Mathematician, Graduate Texts in Mathematics, Springer 5 (1971).

[14] J.W. Milnor. Spin structures on manifolds, Enseignement Math. 9(2) (1963), 198-203.

[15] M. Müger On the structure of modular categories, Proc. Lond. Math. Soc. III. Ser. 87 No. 2, (2003) 291-308.

[16] H. Murakami, T. Ohtsuki, and M. Okada. Invariants of three-manifolds derived from linking matrices of framed links, Osaka J. Math. 29 (1992), 545-572.

[17] B. Ozbagci, and A.I. Stipsicz. Surgery on contact 3-manifolds and Stein surfaces, Springer (2004). 
[18] N. Reshetikhin, and V. Turaev. Invariants of 3-manifolds via link polynomials and quantum groups, Invent. Math. 103 (1991), 547-597.

[19] V.G. Turaev. State sum models in low-dimensional topology, Proc. ICM Kyoto 121 Vol. 1 (1990), 689-698.

[20] V.G. Turaev. Quantum invariants of knots and 3-manifolds. de Gruyter Studies in Math. 18, (1994).

[21] V.G. Turaev. Homotopy Quantum Field Theory, European Mathematical Society, Tracts in Mathematics 10 (2010).

[22] E. Witten. Quantum field theory and the Jones polynomial, Commun. Math. Phys. 121 (1989), 351-399. 
,

$\tilde{\lambda}{ }^{m}$

$\lambda t^{m}$ 